\title{
Variational and Dyson-Schwinger equations of Hamiltonian quantum chromodynamics
}

\author{
Davide Campagnari and Hugo Reinhardt \\ Institut für Theoretische Physik, Universität Tübingen, \\ Auf der Morgenstelle 14, 72076 Tübingen, Germany
}

(Received 10 January 2018; published 23 March 2018)

\begin{abstract}
The variational Hamiltonian approach to quantum chromodynamics in Coulomb gauge is investigated within the framework of the canonical recursive Dyson-Schwinger equations. The dressing of the quark propagator arising from the variationally determined nonperturbative kernels is expanded and renormalized at one-loop order, yielding a chiral condensate compatible with the observations.
\end{abstract}

DOI: 10.1103/PhysRevD.97.054027

\section{INTRODUCTION}

Confinement and spontaneous chiral symmetry breaking are the basic features of quantum chromodynamics (QCD) at ordinary density and temperature. Chiral symmetry breaking is responsible for almost the entire mass of the visible matter in the Universe. Both phenomena originate in the low-energy sector of the theory, where perturbation theory cannot be applied, and are strongly intertwined: lattice calculations show evidence that the deconfinement transition and restoration of chiral symmetry coincide, at least for fermions in the fundamental representation. Thanks to intensive studies both on the lattice [1,2] and in the continuum theory [3-10], we have gained essential insights into the basic features of the QCD vacuum, although a rigorous understanding of both phenomena is still missing. From these studies three pictures have emerged: the dual Meißner effect [11,12], the center vortex scenario [13-16], and the Gribov-Zwanziger picture in Coulomb gauge [17,18]. Both lattice and continuum studies have also shown that these pictures are related [19-21].

The Gribov-Zwanziger picture emerged in the variational Hamiltonian approach to QCD in Coulomb gauge $[8,9]$ : indeed a confining quark potential is found, together with an infrared diverging ghost form factor and gluon energy. A simplified variational calculation [22-25] based on a BCS-type wave functional for the quark sector of QCD, in which the coupling of the quarks to the transverse spatial gluons is neglected, shows that the confining quark potential also triggers chiral symmetry breaking, although the corresponding order parameter, i.e. the chiral

Published by the American Physical Society under the terms of the Creative Commons Attribution 4.0 International license. Further distribution of this work must maintain attribution to the author(s) and the published article's title, journal citation, and DOI. Funded by SCOAP ${ }^{3}$. condensate, turns out to be too small. This model has been phenomenologically improved by using more general twobody interactions [26,27].

In Ref. [28] the variational Hamiltonian approach to Yang-Mills theory in Coulomb gauge $[8,9]$ was extended to full QCD by including the quark-gluon coupling explicitly in the vacuum wave functional. The ansatz for the vacuum wave functional was further extended in Refs. [10,29] by adding a further Dirac structure to the quark-gluon coupling in the trial vacuum wave functional. With this additional Dirac structure the resulting gap equation is free of UV divergences. In the present paper we show that this additional Dirac structure is also crucial to ensure multiplicative renormalizability of the quark propagator. The results of Refs. [10,29] will be retraced here in the framework of the canonical recursive Dyson-Schwinger equations (CRDSEs) $[30,31]$, which, in principle, allows us to go beyond the approximations used in Refs. [10,29] in a systematic way. The use of Dyson-Schwinger equations requires us to formulate the quark sector in terms of Grassmann variables, which turns out to be advantageous over the operator formulation of Fock space used in Refs. [10,28]. Besides reproducing the results of Refs. [10,29] in the framework of the CRDSEs we also investigate analytically the IR behavior of the CRDSE for the quark propagator and determine under which conditions quark confinement is realized. Approximating the full quark-gluon vertex of the CRDSE by its bare counterpart we solve the variational equation and investigate the one-loop renormalization of the quark propagator. From the renormalized quark propagator we calculate the quark condensate.

The structure of this paper is as follows: In Sec. II we reformulate the Hamiltonian approach to QCD within the CRDSEs [31] with the vacuum wave functional proposed in Ref. [10]. In Sec. II A we present the quark vacuum wave functional while in Sec. II B we derive the corresponding CRDSEs for the quark propagator and the quark-gluon 
vertex, by means of which the expectation value of the QCD Hamiltonian is evaluated. In Sec. III we discuss the infrared behavior of the dressing functions of the quark propagator required for confinement. As an illustration of our approach in Sec. IV we keep from the interaction of the quarks only the non-Abelian Coulomb potential resulting in a massive extension of the model considered in Refs. [22,25]. In Sec. V we show how to recover the results of Refs. $[10,29]$ in the present approach by a leading-order skeleton expansion. In Sec. VI we perform a semiperturbative expansion of the quark propagator by using the variational kernels as nonperturbative input and investigate the renormalizability of the quark propagator. In Sec. VII we discuss the relation between the mass function defined in the four-dimensional quark propagator to the mass function of the three-dimensional (equal-time) propagator. Some details concerning the coherent-state description of fermionic states and some explicit expressions are given in the Appendixes.

\section{QCD IN COULOMB GAUGE}

In Coulomb gauge the QCD Hamiltonian reads [32]

$$
\begin{aligned}
H= & \frac{1}{2} \int \mathrm{d}^{3} x J_{A}^{-1} \Pi_{i}^{a}(\mathbf{x}) J_{A} \Pi_{i}^{a}(\mathbf{x})+\frac{1}{2} \int \mathrm{d}^{3} x B_{i}^{a}(\mathbf{x}) B_{i}^{a}(\mathbf{x}) \\
& +\int \mathrm{d}^{3} x \psi^{\dagger}(\mathbf{x})[-\mathrm{i} \boldsymbol{\alpha} \cdot \nabla-g \boldsymbol{\alpha} \cdot \mathbf{A}(\mathbf{x})+\beta m] \psi(\mathbf{x}) \\
& +\frac{g^{2}}{2} \int \mathrm{d}^{3} x \mathrm{~d}^{3} y J_{A}^{-1} \rho^{a}(\mathbf{x}) J_{A} F_{A}^{a b}(\mathbf{x}, \mathbf{y}) \rho^{b}(\mathbf{y}),
\end{aligned}
$$

where $\Pi_{i}^{a}=-\mathrm{i} \delta / \delta A_{i}^{a}$ is the canonical momentum, $B_{i}^{a}$ is the chromomagnetic field, $\psi$ and $\psi^{\dagger}$ are the fermion field operators, $\alpha_{i}$ and $\beta$ are the usual Dirac matrices, $m$ is the bare current quark mass, and $\mathbf{A}=\mathbf{A}^{a} t^{a}$ are the (transverse) gauge fields with $t^{a}$ being the Hermitian generators of the $\mathfrak{g} \mathfrak{t}\left(N_{c}\right)$ algebra. The last term in Eq. (1) is the so-called Coulomb term: it describes the interaction of the color charge density

$$
\rho^{a}(\mathbf{x})=\psi^{\dagger}(\mathbf{x}) t^{a} \psi(\mathbf{x})+f^{a b c} A_{i}^{b}(\mathbf{x}) \frac{\delta}{\mathrm{i} \delta A_{i}^{c}(\mathbf{x})}
$$

through the Coulomb kernel

$$
F_{A}^{a b}(\mathbf{x}, \mathbf{y})=\int \mathrm{d}^{3} z G_{A}^{a c}(\mathbf{x}, \mathbf{z})\left(-\nabla_{z}^{2}\right) G_{A}^{c b}(\mathbf{z}, \mathbf{y}),
$$

where

$$
G_{A}^{-1}(\mathbf{x}, \mathbf{y})=\left(-\delta^{a b} \nabla_{x}^{2}-g f^{a c b} A_{i}^{c}(\mathbf{x}) \partial_{i}^{x}\right) \delta(\mathbf{x}-\mathbf{y})
$$

is the Faddeev-Popov operator of Coulomb gauge with $f^{a c b}$ being the structure constants of the $\mathfrak{g} \mathfrak{t}\left(N_{c}\right)$ algebra. Finally, $J_{A}=\operatorname{Det} G_{A}^{-1}$ is the Faddeev-Popov determinant of Coulomb gauge.

\section{A. The vacuum wave functional}

In the coherent-state description of the fermionic Fock space introduced in Ref. [31] (see Appendix A) a physical state $|\Psi\rangle$ is described by a functional

$$
\left\langle\xi, \xi^{\dagger}, A \mid \Psi\right\rangle=\Psi\left[\xi_{+}^{\dagger}, \xi_{-}, A\right]
$$

of the gauge fields $A_{i}$ and of the spinor-valued Grassmann fields

$$
\xi_{ \pm}(1)=\Lambda_{ \pm}(1,2) \xi(2),
$$

where

$$
\begin{aligned}
\Lambda_{ \pm}(1,2) & =\int \frac{\mathrm{d}^{3} p}{(2 \pi)^{3}} \mathrm{e}^{\mathrm{i} \cdot\left(\mathbf{x}_{1}-\mathbf{x}_{2}\right)} \Lambda_{ \pm}(\mathbf{p}), \\
\Lambda_{ \pm}(\mathbf{p}) & =\frac{1}{2} \pm \frac{\boldsymbol{\alpha} \cdot \mathbf{p}+\beta m}{2 \sqrt{\mathbf{p}^{2}+m^{2}}}
\end{aligned}
$$

are the projectors onto positive/negative energy eigenstates of the free Dirac operator

$$
h_{0}(\mathbf{p})=\boldsymbol{\alpha} \cdot \mathbf{p}+\beta m .
$$

In coordinate space we employ a notation where a numerical index stands collectively for the spatial coordinate as well as for the color and Lorentz indices. A repeated numerical index like in Eq. (4) implies integration over the spatial coordinate and summation over the discrete indices. Matrix elements of an operator $O$ between physical states $\Phi$ and $\Psi$ are given by the functional integral

$$
\begin{aligned}
\left\langle\Phi\left|O\left[A, \Pi, \psi, \psi^{\dagger}\right]\right| \Psi\right\rangle= & \int \mathcal{D} \xi \mathcal{D} \xi^{\dagger} \mathcal{D} A J_{A} \mathrm{e}^{-\mu} \Phi^{*}\left[\xi_{+}^{\dagger}, \xi_{-}, A\right] \\
& \times O\left[A,-\mathrm{i} \frac{\delta}{\delta A}, \xi_{-}+\frac{\delta}{\delta \xi_{+}^{\dagger}}, \xi_{+}^{\dagger}+\frac{\delta}{\delta \xi_{-}}\right] \\
& \times \Psi\left[\xi_{+}^{\dagger}, \xi_{-}, A\right],
\end{aligned}
$$

where

$$
\mu=\xi^{\dagger}(1) S_{0}(1,2) \xi(2)=\int \frac{\mathrm{d}^{3} p}{(2 \pi)^{3}} \xi^{\dagger}(\mathbf{p}) S_{0}(\mathbf{p}) \xi(\mathbf{p}),
$$

is the integration measure of the fermionic coherent states, which involves the bare quark propagator

$S_{0}(\mathbf{p})=\frac{h_{0}(\mathbf{p})}{2 E_{\mathbf{p}}}=\frac{\boldsymbol{\alpha} \cdot \mathbf{p}+\beta m}{2 E_{\mathbf{p}}}, \quad E_{\mathbf{p}}=\sqrt{\mathbf{p}^{2}+m^{2}}$.

For the vacuum wave functional of QCD we take the ansatz 


$$
\Psi\left[A, \xi_{+}^{\dagger}, \xi_{-}\right] \propto \exp \left\{-\frac{1}{2} S_{A}[A]-S_{f}\left[\xi_{+}^{\dagger}, \xi_{-}, A\right]\right\}
$$

where $S_{A}$ and $S_{f}$ define, respectively, the wave functionals of pure Yang-Mills theory and of the quarks interacting with the gluons. We choose the latter in the form

$$
\begin{aligned}
S_{f}\left[\xi_{+}^{\dagger}, \xi_{-}, A\right]= & \xi_{+}^{\dagger}(1)\left[K_{0}(1,2)+K(1,2 ; 3) A(3)\right] \xi_{-}(2) \\
= & \xi^{\dagger}\left(1^{\prime}\right) \Lambda_{+}\left(1^{\prime}, 1\right)\left[K_{0}(1,2)+K(1,2 ; 3) A(3)\right] \\
& \times \Lambda_{-}\left(2,2^{\prime}\right) \xi\left(2^{\prime}\right)
\end{aligned}
$$

where $K_{0}$ and $K$ are variational kernels, whose form will be specified in more detail later.

Once the functional derivatives in Eq. (7) are taken, expectation values of operators boil down to quantum averages of functionals of the fields

$$
\left\langle f\left[\xi^{\dagger}, \xi, A\right]\right\rangle=\int \mathcal{D} \xi^{\dagger} \mathcal{D} \xi \mathcal{D} A J_{A} \mathrm{e}^{-S} f\left[\xi^{\dagger}, \xi, A\right]
$$

with an "action"

$$
S=S_{A}+S_{f}+S_{f}^{*}+\mu .
$$

This equivalence between expectation values in the Hamiltonian approach and quantum averages in the functional integral formulation of a Euclidean field theory in $d=3$ dimensions is the basis for the Dyson-Schwinger approach $[30,31]$ employed in this work. With the help of familiar Dyson-Schwinger techniques the various oneparticle irreducible equal-time Green functions of the Hamiltonian approach can be related to the kernels occurring in the action equation (11), i.e. in the vacuum wave functional equation (9), by means of an infinite tower of integral equations. These are named CRDSEs in order to make clear that, while they look like standard DSEs, their physical content is somewhat different. (The bare $n$-point vertices are not given by the action of the theory but by variational kernels of the vacuum wave functionals.)

Notice that the variational kernels $K_{0}$ and $K_{i}$ in Eq. (10) enter the action equation (11) (and therefore the CRDSEs) only in the combinations

$$
\begin{aligned}
\bar{\gamma}(1,2)= & \Lambda_{+}\left(1,1^{\prime}\right) K_{0}\left(1^{\prime}, 2^{\prime}\right) \Lambda_{-}\left(2^{\prime}, 2\right) \\
& +\Lambda_{-}\left(1,1^{\prime}\right) K_{0}^{\dagger}\left(1^{\prime}, 2^{\prime}\right) \Lambda_{+}\left(2^{\prime}, 2\right)
\end{aligned}
$$

and

$$
\begin{aligned}
\bar{\Gamma}_{0}(1,2 ; 3)= & \Lambda_{+}\left(1,1^{\prime}\right) K\left(1^{\prime}, 2^{\prime} ; 3\right) \Lambda_{-}\left(2^{\prime}, 2\right) \\
& +\Lambda_{-}\left(1,1^{\prime}\right) K^{\dagger}\left(1^{\prime}, 2^{\prime} ; 3\right) \Lambda_{+}\left(2^{\prime}, 2\right) .
\end{aligned}
$$

In the following we will refer to $\bar{\gamma}$ as the biquark kernel, and to $\bar{\Gamma}_{0}$ as the bare quark-gluon vertex. ${ }^{1}$

The choice of the variational kernels in Eq. (10) is subject to a restriction: the vacuum wave functional must be invariant under global color rotations. These are generated by the total charge operator

$$
Q^{a}=\int \mathrm{d}^{3} x \rho^{a}(\mathbf{x})
$$

i.e. the spatial integral of the color charge density Eq. (2). Invariance under global color rotations generated by $Q^{a}$ implies that the wave functional equation (9) (or, equivalently, the quantities $S_{A}$ and $S_{f}$ occurring in its exponent) must be annihilated by $Q^{a}$, which leads to the condition

$$
\begin{aligned}
Q^{a} S_{f}= & \int \mathrm{d}^{3} x \mathrm{~d}^{3} y \xi_{+}^{\dagger}(\mathbf{x}) \\
& \times\left\{\left[t^{a}, K_{0}(\mathbf{x}, \mathbf{y})\right]+\int \mathrm{d}^{3} z\left(\left[t^{a}, K_{i}^{b}(\mathbf{x}, \mathbf{y} ; \mathbf{z})\right]\right.\right. \\
& \left.\left.-\mathrm{i} f^{a b c} K_{i}^{c}(\mathbf{x}, \mathbf{y} ; \mathbf{z})\right) A_{i}^{b}(\mathbf{z})\right\} \xi_{-}(\mathbf{y}) \stackrel{!}{=} 0 .
\end{aligned}
$$

In order to satisfy this condition the variational kernels should obey the color structure $K_{0} \sim \mathbb{1}$ and $K_{i}^{a} \sim t^{a}$. Furthermore, since the $\Lambda_{ \pm}$are orthogonal projectors [see Eq. (5)] it is obvious from Eq. (12) that the variational kernel $K_{0}$ must possess nontrivial Dirac structures.

In principle, $K_{0}$ could have the general form

$$
K_{0}(\mathbf{p})=\beta s_{1}(\mathbf{p})+\boldsymbol{\alpha} \cdot \mathbf{p} s_{2}(\mathbf{p})+\beta \boldsymbol{\alpha} \cdot \mathbf{p} s_{3}(\mathbf{p})
$$

with complex scalar functions $s_{1}, s_{2}, s_{3}$. The resulting biquark kernel equation (12) becomes in momentum space

$$
\begin{aligned}
\bar{\gamma}(\mathbf{p})= & \frac{\beta \mathbf{p}^{2}-m \boldsymbol{\alpha} \cdot \mathbf{p}}{E_{\mathbf{p}}^{2}} \Re\left\{s_{1}(\mathbf{p})-m s_{2}(\mathbf{p})-E_{\mathbf{p}} s_{3}(\mathbf{p})\right\} \\
& -\frac{\mathrm{i} \beta \boldsymbol{\alpha} \cdot \mathbf{p}}{E_{\mathbf{p}}} \Im\left\{s_{1}(\mathbf{p})-m s_{2}(\mathbf{p})-E_{\mathbf{p}} s_{3}(\mathbf{p})\right\} .
\end{aligned}
$$

As one observes, the complex kernels $s_{1}, s_{2}$, and $s_{3}$ enter the biquark kernel $\bar{\gamma}$ (and therefore all vacuum expectation values of observables as well as the CRDSEs) never individually but only in the combination

$$
s_{1}(\mathbf{p})-m s_{2}(\mathbf{p})-E_{\mathbf{p}} s_{3}(\mathbf{p}) .
$$

It is hence sufficient to consider only one of them; moreover, in the chiral limit $m=0$ the scalar kernel $s_{2}$ drops out.

\footnotetext{
${ }^{1}$ The bare quark-gluon vertex $\bar{\Gamma}_{0}$ entering the vacuum wave functional equation (9) should be distinguished from the quarkgluon coupling in the QCD Hamiltonian equation (1).
} 
Therefore, the general ansatz equation (14) is not necessary. Instead the relevant physics can be captured by the much simpler choice

$$
K_{0}(\mathbf{p})=\beta s(\mathbf{p})
$$

which leads to the biquark kernel [Eq. (12)]

$$
\bar{\gamma}(\mathbf{p})=-\boldsymbol{\alpha} \cdot \hat{\mathbf{p}} \frac{m|\mathbf{p}|}{E_{\mathbf{p}}^{2}} \Re s(\mathbf{p})+\beta \frac{\mathbf{p}^{2}}{E_{\mathbf{p}}^{2}} \Re s(\mathbf{p})-\mathrm{i} \beta \boldsymbol{\alpha} \cdot \hat{\mathbf{p}} \frac{|\mathbf{p}|}{E_{\mathbf{p}}} \Im s(\mathbf{p}) .
$$

For the vector kernel we choose the ansatz [10]

$$
\begin{aligned}
K_{i}^{m n, a}(\mathbf{p}, \mathbf{q} ; \mathbf{k})= & g t_{m n}^{a}\left[\alpha_{i} V(\mathbf{p}, \mathbf{q})+\beta \alpha_{i} W(\mathbf{p}, \mathbf{q})\right] \\
& \times(2 \pi)^{3} \delta(\mathbf{p}+\mathbf{q}+\mathbf{k}),
\end{aligned}
$$

where $V$, and $W$ are variational kernels: For simplicity we write only their dependence on the quark-anti-quark momenta, as momentum conservation implicitly fixes the gluon momentum. Note that the vectorial character of the quark-gluon coupling is entirely given by the Dirac matrix $\alpha_{i}$, i.e. the variational kernels $V$ and $W$ are scalar functions which may depend only on $\mathbf{p}^{2}, \mathbf{q}^{2}$, and $\mathbf{p} \cdot \mathbf{q}$, implying e.g. $V(-\mathbf{p},-\mathbf{q})=V(\mathbf{p}, \mathbf{q})$. The bare quark-gluon vertex equation (13) becomes with Eq. (17) in the chiral limit

$$
\begin{aligned}
\bar{\Gamma}_{0, i}^{m n, a}(\mathbf{p}, \mathbf{q} ; \mathbf{k}) \\
=t_{m n}^{a} \frac{g}{4}\left[(1+\boldsymbol{\alpha} \cdot \hat{\mathbf{p}})\left[V(\mathbf{p}, \mathbf{q}) \alpha_{i}+W(\mathbf{p}, \mathbf{q}) \beta \alpha_{i}\right](1+\boldsymbol{\alpha} \cdot \hat{\mathbf{q}})\right. \\
\left.\quad+(1-\boldsymbol{\alpha} \cdot \hat{\mathbf{p}})\left[V^{*}(\mathbf{q}, \mathbf{p}) \alpha_{i}-W^{*}(\mathbf{q}, \mathbf{p}) \beta \alpha_{i}\right](1-\boldsymbol{\alpha} \cdot \hat{\mathbf{q}})\right] \\
\quad \times(2 \pi)^{3} \delta(\mathbf{p}+\mathbf{q}+\mathbf{k}) .
\end{aligned}
$$

When both vector kernels are omitted, $V(\mathbf{p}, \mathbf{q})=0=$ $W(\mathbf{p}, \mathbf{q})$, the wave functional equation (9) reduces to the BCS-type wave functional used in Refs. [22,25,27], while keeping only $V$ corresponds to the choice of Refs. [28]. The above ansatz for the fermionic wave functional defined by Eqs. (9), (10), (15), and (17) was also chosen in Refs. [10,29], where the QCD variational principle was formulated in the ordinary operator language of second quantization, avoiding the introduction of Grassmann fields. As shown in Ref. [29] this ansatz has the advantage that all UV divergences cancel in the quark gap equation.

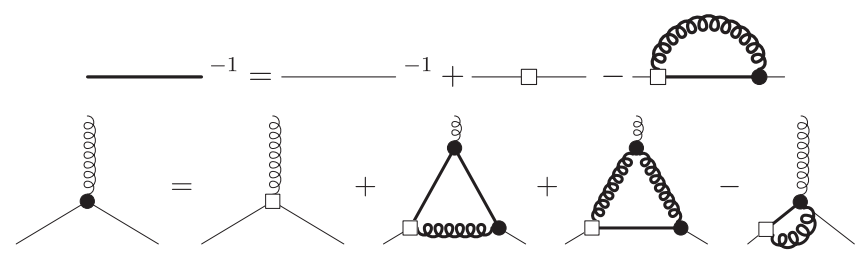

FIG. 1. Diagrammatic representation of the CRDSEs for the quark propagator [top, Eq. (19)] and for the quark-gluon vertex (bottom). Full lines and filled dots represent, respectively, dressed propagators and vertices. The line with an empty square stands for the biquark kernel $\bar{\gamma}$ [Eq. (16)]; the vertex with a square box represents the bare quark-gluon vertex [Eq. (18)].

\section{B. Quark propagator and quark-gluon vertex CRDSEs}

As shown in Refs. [30,31] the formal equivalence between expectation values in the Hamiltonian approach and quantum averages of a Euclidean field theory can be used to write down DSE-like equations, referred to as CRDSEs to express the $n$-point functions by the variational kernels of the vacuum wave functional. The CRDSE for the fermion propagator

$$
Q(1,2)=\left\langle\xi(1) \xi^{\dagger}(2)\right\rangle
$$

reads

$$
\begin{aligned}
Q^{-1}(1,2)= & Q_{0}^{-1}(1,2)+\bar{\gamma}(1,2) \\
& -\bar{\Gamma}_{0}(1,3 ; 4) Q\left(3,3^{\prime}\right) D\left(4,4^{\prime}\right) \bar{\Gamma}\left(3^{\prime}, 2 ; 4^{\prime}\right),
\end{aligned}
$$

where

$$
Q_{0}(1,2)=\Lambda_{+}(1,2)-\Lambda_{-}(1,2)
$$

is the bare fermion propagator,

$$
D(1,2)=\langle A(1) A(2)\rangle
$$

is the gluon propagator, and $\bar{\Gamma}$ is the full quark-gluon vertex defined by

$\left\langle\xi(1) \xi^{\dagger}(2) A(3)\right\rangle=-Q\left(1,1^{\prime}\right) \bar{\Gamma}\left(1^{\prime}, 2^{\prime} ; 3^{\prime}\right) Q\left(2^{\prime}, 2\right) D\left(3^{\prime}, 3\right)$.

The latter also obeys a CRDSE, which is represented diagrammatically together with Eq. (19) in Fig. 1. The explicit form is not relevant for the present work but the first term on the right-hand side is given indeed by $\bar{\Gamma}_{0}$ [Eq. (18)], thus justifying its interpretation as bare quarkgluon vertex.

Equation (19) may be conveniently written in momentum space: with the explicit form [Eq. (16)] of the biquark kernel we obtain 


$$
\begin{aligned}
{\left[Q^{m n}(\mathbf{p})\right]^{-1}=} & \delta^{m n} \frac{\boldsymbol{\alpha} \cdot \mathbf{p}+\beta m}{E_{\mathbf{p}}}+\delta^{m n}\left(-\boldsymbol{\alpha} \cdot \mathbf{p} \frac{m}{E_{\mathbf{p}}^{2}} \Re s(\mathbf{p})+\beta \frac{\mathbf{p}^{2}}{E_{\mathbf{p}}^{2}} \Re s(\mathbf{p})-\mathrm{i} \beta \boldsymbol{\alpha} \cdot \mathbf{p} \frac{1}{E_{\mathbf{p}}} \Im s(\mathbf{p})\right) \\
& -\int \frac{\mathrm{d}^{3} q}{(2 \pi)^{3}} \bar{\Gamma}_{0, i}^{m k, a}(\mathbf{p},-\mathbf{q} ; \mathbf{q}-\mathbf{p}) Q(\mathbf{q}) D_{i j}(\mathbf{p}-\mathbf{q}) \bar{\Gamma}_{j}^{k n, a}(\mathbf{q},-\mathbf{p} ; \mathbf{p}-\mathbf{q}) .
\end{aligned}
$$

For the inverse quark propagator, which we assume to be color diagonal, we must consider in principle the following Dirac structure:

$Q^{-1}(\mathbf{p})=A(\mathbf{p}) \boldsymbol{\alpha} \cdot \hat{\mathbf{p}}+\beta B(\mathbf{p})-\mathrm{i} \beta \boldsymbol{\alpha} \cdot \hat{\mathbf{p}} C(\mathbf{p})+D(\mathbf{p})$,

which can be inverted to give
$Q(\mathbf{p})=\frac{A(\mathbf{p}) \boldsymbol{\alpha} \cdot \hat{\mathbf{p}}+\beta B(\mathbf{p})-\mathrm{i} \beta \boldsymbol{\alpha} \cdot \hat{\mathbf{p}} C(\mathbf{p})-D(\mathbf{p})}{A^{2}(\mathbf{p})+B^{2}(\mathbf{p})+C^{2}(\mathbf{p})-D^{2}(\mathbf{p})}$.

From the CRDSE (22) we obtain the following system of coupled equations for the dressing functions [Eq. (23)] of the quark propagator

$$
\begin{aligned}
& \left.A(\mathbf{p})=\frac{|\mathbf{p}|}{E_{\mathbf{p}}}\left(1-\frac{m}{E_{\mathbf{p}}} \Re s \mathbf{p}\right)\right)-\frac{1}{4 N_{c}} \int \frac{\mathrm{d}^{3} q}{(2 \pi)^{3}} \operatorname{tr}\left[\boldsymbol{\alpha} \cdot \hat{\mathbf{p}} \bar{\Gamma}_{0, i}^{m n, a}(\mathbf{p},-\mathbf{q} ; \mathbf{q}-\mathbf{p}) Q(\mathbf{q}) D_{i j}(\mathbf{p}-\mathbf{q}) \bar{\Gamma}_{j}^{n m, a}(\mathbf{q},-\mathbf{p} ; \mathbf{p}-\mathbf{q})\right], \\
& B(\mathbf{p})=\frac{m}{E_{\mathbf{p}}}+\frac{\mathbf{p}^{2}}{E_{\mathbf{p}}^{2}} \Re s(\mathbf{p})-\frac{1}{4 N_{c}} \int \frac{\mathrm{d}^{3} q}{(2 \pi)^{3}} \operatorname{tr}\left[\beta \bar{\Gamma}_{0, i}^{m n, a}(\mathbf{p},-\mathbf{q} ; \mathbf{q}-\mathbf{p}) Q(\mathbf{q}) D_{i j}(\mathbf{p}-\mathbf{q}) \bar{\Gamma}_{j}^{n m, a}(\mathbf{q},-\mathbf{p} ; \mathbf{p}-\mathbf{q})\right], \\
& C(\mathbf{p})=\frac{|\mathbf{p}|}{E_{\mathbf{p}}} \Im s(\mathbf{p})-\frac{1}{4 N_{c}} \int \frac{\mathrm{d}^{3} q}{(2 \pi)^{3}} \operatorname{tr}\left[-\mathrm{i} \beta \boldsymbol{\alpha} \cdot \hat{\mathbf{p}} \bar{\Gamma}_{0, i}^{m n, a}(\mathbf{p},-\mathbf{q} ; \mathbf{q}-\mathbf{p}) Q(\mathbf{q}) D_{i j}(\mathbf{p}-\mathbf{q}) \bar{\Gamma}_{j}^{n m, a}(\mathbf{q},-\mathbf{p} ; \mathbf{p}-\mathbf{q})\right], \\
& D(\mathbf{p})=-\frac{1}{4 N_{c}} \int \frac{\mathrm{d}^{3} q}{(2 \pi)^{3}} \operatorname{tr}\left[\bar{\Gamma}_{0, i}^{m n, a}(\mathbf{p},-\mathbf{q} ; \mathbf{q}-\mathbf{p}) Q(\mathbf{q}) D_{i j}(\mathbf{p}-\mathbf{q}) \bar{\Gamma}_{j}^{n m, a}(\mathbf{q},-\mathbf{p} ; \mathbf{p}-\mathbf{q})\right],
\end{aligned}
$$

where

$$
D_{i j}(\mathbf{p}) \equiv \frac{t_{i j}(\mathbf{p})}{2 \Omega(\mathbf{p})}, \quad t_{i j}(\mathbf{p})=\delta_{i j}-\frac{p_{i} p_{j}}{\mathbf{p}^{2}}
$$

is the gluon propagator equation (20), conveniently parametrized in terms of the quasigluon energy $\Omega(\mathbf{p})$.

At this point it should be mentioned that the fermion propagator $Q$ is not the physical quark propagator, which in the Hamiltonian approach is defined by

$$
S(1,2)=\frac{1}{2}\left\langle\left[\psi(1), \psi^{\dagger}(2)\right]\right\rangle .
$$

The commutator arises from the equal-time limit of the time-ordered operator product in the full time-dependent theory. By means of Eq. (7) one can show (for details see Ref. [31]) that the quark propagator $S$ and the propagator $Q$ are related by

$$
S(\mathbf{p})=Q(\mathbf{p})-S_{0}(\mathbf{p})
$$

with $S_{0}(\mathbf{p})$ being the free quark propagator, Eq. (8). As long as no confusion is possible we will keep referring indiscriminately to both $S(\mathbf{p})$ and $Q(\mathbf{p})$ as quark propagator.

\section{The QCD vacuum energy density}

The vacuum expectation value of the QCD Hamiltonian has been evaluated in Ref. [31], to which we refer the reader for the details of the calculation; here we will merely quote the relevant contributions to the energy density $e \equiv$ $\langle H\rangle /\left(V \cdot N_{c}\right)$ in momentum space. The Dirac Hamiltonian [second line in Eq. (1)] yields

$$
\begin{aligned}
e_{\mathrm{D}}= & -\int \frac{\mathrm{d}^{3} q}{(2 \pi)^{3}} \operatorname{tr}[(\boldsymbol{\alpha} \cdot \mathbf{q}+\beta m) Q(\mathbf{q})] \\
& -g C_{F} \int \frac{\mathrm{d}^{3} q}{(2 \pi)^{3}} \frac{\mathrm{d}^{3} \ell}{(2 \pi)^{3}} \\
& \times D_{i j}(\mathbf{q}+\boldsymbol{\ell}) \operatorname{tr}\left[\alpha_{i} Q(\mathbf{q}) \bar{\Gamma}_{j}(\mathbf{q}, \boldsymbol{\ell}) Q(-\boldsymbol{\ell})\right] \\
\equiv & e_{\mathrm{D}}^{(0)}+e_{\mathrm{D}}^{(1)},
\end{aligned}
$$

where $C_{F}=\left(N_{c}^{2}-1\right) /\left(2 N_{c}\right)$ is the quadratic Casimir invariant of the fundamental representation of the $\mathfrak{g} \mathfrak{t}\left(N_{\mathrm{c}}\right)$ algebra. The fermionic contribution to the kinetic energy of the gluons [first term on the right-hand side of Eq. (1)] is given by 


$$
\begin{aligned}
e_{E}^{q}= & -\frac{C_{F}}{8} \int \frac{\mathrm{d}^{3} q}{(2 \pi)^{3}} \frac{\mathrm{d}^{3} \ell}{(2 \pi)^{3}} t_{i j}(\mathbf{q}+\boldsymbol{\ell}) \operatorname{tr}\left\{\bar{\Gamma}_{0, i}(\mathbf{q},-\boldsymbol{\ell}) Q(\boldsymbol{\ell}) \bar{\Gamma}_{j}(\boldsymbol{\ell},-\mathbf{q}) Q(\mathbf{q})\right. \\
& \left.-Q_{0}(\mathbf{q}) \bar{\Gamma}_{0, i}(\mathbf{q},-\boldsymbol{\ell}) Q(\boldsymbol{\ell}) Q_{0}(\boldsymbol{\ell}) \bar{\Gamma}_{0, j}(\boldsymbol{\ell},-\mathbf{q}) Q(\mathbf{q})\right\} .
\end{aligned}
$$

For simplicity, in Eqs. (27) and (28) we have omitted the dependence of the vertex functions on the gluon momentum, which follows from the fermionic momenta kept in the above equations by momentum conservation. Furthermore, we have assumed that the propagators are color diagonal and that the color structure of the full quark-gluon vertex is given by the generator $t^{a}$ as for the bare vertex. Finally, the Coulomb interaction of the fermionic charges reads

$$
\begin{aligned}
e_{\mathrm{C}}^{q q} \simeq & -g^{2} \frac{C_{F}}{2} \int \frac{\mathrm{d}^{3} q}{(2 \pi)^{3}} \frac{\mathrm{d}^{3} \ell}{(2 \pi)^{3}} F(\mathbf{q}-\boldsymbol{\ell}) \\
& \times \operatorname{tr}\left\{\left[Q(\boldsymbol{\ell})-\frac{1}{2} Q_{0}(\boldsymbol{\ell})\right]\left[Q(\mathbf{q})-\frac{1}{2} Q_{0}(\mathbf{q})\right]-\frac{1}{4}\right\} .
\end{aligned}
$$

Here, $F(\mathbf{p})$ is the expectation value of the Coulomb kernel equation (3), which in the following calculations will be approximated by the simple form [9]:

$$
g^{2} F(\mathbf{p})=\frac{8 \pi \sigma_{\mathrm{C}}}{\mathbf{p}^{4}}+\frac{g^{2}}{\mathbf{p}^{2}}
$$

with $\sigma_{\mathrm{C}}$ being the Coulomb string tension. This form nicely fits the Coulomb potential found from the variational solution of the Yang-Mills sector [9].

Since the expectation value $e_{\mathrm{D}}^{(0)}$ of the single-particle Hamiltonian [first term in Eq. (27)] and the Coulomb interaction equation (29) do not depend on the full quarkgluon vertex, the Dirac traces can be worked out explicitly, yielding respectively

$$
e_{\mathrm{D}}^{(0)}=-4 \int \frac{\mathrm{d}^{3} q}{(2 \pi)^{3}} \frac{|\mathbf{q}| A(\mathbf{q})+m B(\mathbf{q})}{\Delta(\mathbf{q})}
$$

and

$$
\begin{aligned}
e_{\mathrm{C}}^{q q}= & -g^{2} \frac{C_{F}}{2} \int \frac{\mathrm{d}^{3} q}{(2 \pi)^{3}} \frac{\mathrm{d}^{3} \ell}{(2 \pi)^{3}} \frac{F(\mathbf{q}-\boldsymbol{\ell})}{\Delta(\mathbf{q}) \Delta(\boldsymbol{\ell})} \\
& \times\{4[B(\mathbf{q}) B(\boldsymbol{\ell})+D(\mathbf{q}) D(\boldsymbol{\ell})]-\Delta(\mathbf{q}) \Delta(\boldsymbol{\ell}) \\
& +\hat{\mathbf{q}} \cdot \hat{\boldsymbol{\ell}}[4 C(\mathbf{q}) C(\boldsymbol{\ell}) \\
& +(2 A(\mathbf{q})-\Delta(\mathbf{q}))(2 A(\boldsymbol{\ell})-\Delta(\boldsymbol{\ell}))]\},
\end{aligned}
$$

where we have introduced the abbreviation

$$
\Delta(\mathbf{q})=A^{2}(\mathbf{q})+B^{2}(\mathbf{q})+C^{2}(\mathbf{q})-D^{2}(\mathbf{q})
$$

for the denominator of the quark propagator equation (24).

\section{INFRARED BEHAVIOR OF THE DRESSING FUNCTIONS}

Before we proceed to derive the equations of motion of our variational approach by minimizing the energy density with respect to the variational kernels, we discuss here which conditions the dressing functions $A(\mathbf{p}), \ldots, D(\mathbf{p})$ of the quark propagator equation (23) must satisfy in order to guarantee confinement and chiral symmetry breaking. For given variational kernels of the wave functional these dressing functions are determined by the quark propagator CRDSE (25), while the variational kernels themselves are determined by minimizing the energy density.

For simplicity we assume that the vector kernels $V(\mathbf{p}, \mathbf{q})$ and $W(\mathbf{p}, \mathbf{q})$ are real and symmetric, and that the scalar kernel $s(\mathbf{p})$ is real (we can always restrict our variational ansatz to these class of kernels): then, consistent solutions with $D(\mathbf{p})=0$ and $C(\mathbf{p})=0$ exist, see Eq. (B1) below. We will furthermore restrict our considerations to chiral quarks, $m=0$.

As we have shown in Sec. II B, the physical quark propagator $S$ is related to the propagator $Q$ of the Grassmann fields by Eq. (26) and can be expressed through the dressing functions $A$ and $B$ as

$$
\begin{aligned}
S(\mathbf{p}) & =Q(\mathbf{p})-S_{0}(\mathbf{p}) \\
& =\frac{\left[A_{p}\left(2-A_{p}\right)-B_{p}^{2}\right] \boldsymbol{\alpha} \cdot \hat{\mathbf{p}}+2 B_{p} \beta}{2\left(A_{p}^{2}+B_{p}^{2}\right)} .
\end{aligned}
$$

In order to prevent the notation from becoming excessively cluttered we have expressed the momentum dependence of the dressing functions through a subscript.

Inspired by the form of the bare quark propagator [Eq. (8)] we define the running mass $M_{p}$ and the and the quark dressing function $Z_{p}$ by

$$
S(\mathbf{p})=Z_{p} \frac{\boldsymbol{\alpha} \cdot \mathbf{p}+\beta M_{p}}{2 \mathcal{E}_{p}}, \quad \mathcal{E}_{p}=\sqrt{p^{2}+M_{p}^{2}} .
$$

From Eqs. (34) and (35) we obtain

$$
\begin{aligned}
M_{p} & =\frac{2 p B_{p}}{A_{p}\left(2-A_{p}\right)-B_{p}^{2}}, \\
Z_{p} & =\frac{\sqrt{\left[A_{p}\left(2-A_{p}\right)-B_{p}^{2}\right]^{2}+4 B_{p}^{2}}}{A_{p}^{2}+B_{p}^{2}},
\end{aligned}
$$

where $p=|\mathbf{p}|$. These equations can be inverted to express the dressing functions $A_{p}$ and $B_{p}$ in terms of $M_{p}$ and $Z_{p}$ as 
$A_{p}=\frac{2\left(\mathcal{E}_{p}+p Z_{p}\right)}{\mathcal{E}_{p}\left(1+Z_{p}^{2}\right)+2 p Z_{p}}, \quad B_{p}=\frac{2 M_{p} Z_{p}}{\mathcal{E}_{p}\left(1+Z_{p}^{2}\right)+2 p Z_{p}}$.

Note that the approximation $A_{p}=1$ is equivalent to $Z_{p}=1$. We will now exploit these relations to investigate the IR behavior of the dressing functions $A_{p}$ and $B_{p}$.

An IR finite mass function $M(p=0) \equiv M_{0} \neq 0$ is an indicator of chiral symmetry breaking. Therefore we investigate now which conditions the functions $A_{p}$ and $B_{p}$ must fulfill at vanishing momentum so that $M_{0} \neq 0$. From Eq. (36) follows immediately that an IR diverging $B_{p}$ and an IR finite $A_{p}$ would give rise to a vanishing (negative) mass function. The dressing function $B_{p}$ must therefore have a finite IR limit $B_{0}$. Furthermore, from the first equation in (36) it follows that the dressing function $A_{p}$ must also have a finite IR limit $A_{0}$ satisfying the condition

$$
A_{0}\left(2-A_{0}\right)=B_{0}^{2}
$$

Hence for real $B_{0}$ and $A_{0}$ we find that $A_{0} \in[0,2]$. From the second expression in Eq. (36) we find in the limit of vanishing momentum assuming that Eq. (38) holds

$$
Z_{0}=\sqrt{\frac{2-A_{0}}{A_{0}}} .
$$

Like Eq. (38), the right-hand side of Eq. (39) is well defined only for $A_{0} \in[0,2]$. An infrared suppressed propagator $Z_{0}<1$ requires $A_{0}>1$, and an IR vanishing quark propagator requires $A_{0}=2$, which in view of Eq. (38) implies $B_{0}=0$. For the mass function to be still nonvanishing in the IR, the dressing function $A$ should have zero slope at vanishing momentum, as it can be seen by Taylor expanding equation (36).

From this IR analysis there emerges a possible GribovZwanziger-like scenario which includes both confinement and chiral symmetry breaking: an IR vanishing dressing function $B_{p}$ and a dressing function $A_{p}$ satisfying $A(0)=2$ and $A^{\prime}(0)=0$ yield an IR finite running mass (i.e. spontaneous breaking of chiral symmetry) and an IR vanishing (i.e. confined) quark propagator. The same conclusions follow of course from Eq. (37) taken at zero momentum

$$
A_{0}=\frac{2}{1+Z_{0}^{2}}, \quad B_{p}=\frac{2 Z_{0}}{1+Z_{0}^{2}}
$$

For an infrared vanishing quark propagator, $Z_{0}=0$, we find immediately $A_{0}=2$ and $B_{0}=0$.

The above results are based in the analysis of the unrenormalized CRDSEs and may hence change after renormalization. However, the renormalization affects mostly the UV behavior.

\section{MASSIVE ADLER-DAVIS MODEL}

To make contact with previous work and for the sake of illustration, in the present section let us neglect the quarkgluon coupling in the QCD Hamiltonian and consider the quark sector only. The remaining contributions to the energy density are therefore Eqs. (31) and (32). If we neglect the coupling of the quarks to the transverse (spatial) gluons in the vacuum wave functional equation (9), (10), i.e. $V=0=W$, the bare quark-gluon vertex equation (18) vanishes, $\bar{\Gamma}_{0}=0$. Furthermore, if the scalar kernel $s_{p}$ is real both dressing functions $C_{p}$ and $D_{p}$ vanish identically. Then the energy density reduces to

$$
\begin{aligned}
e_{\mathrm{AD}}= & -4 \int \frac{\mathrm{d}^{3} q}{(2 \pi)^{3}} \frac{|\mathbf{q}| A_{q}+m B_{q}}{\Delta_{q}} \\
& -g^{2} \frac{C_{F}}{2} \int \frac{\mathrm{d}^{3} q}{(2 \pi)^{3}} \frac{\mathrm{d}^{3} \ell}{(2 \pi)^{3}} \frac{F(\mathbf{q}-\boldsymbol{\ell})}{\Delta_{q} \Delta_{\ell}} \\
& \times\left\{4 B_{q} B_{\ell}+\hat{\mathbf{q}} \cdot \hat{\ell}\left[A_{q}\left(2-A_{q}\right)-B_{q}^{2}\right]\right. \\
& \left.\times\left[A_{\ell}\left(2-A_{\ell}\right)-B_{\ell}^{2}\right]\right\},
\end{aligned}
$$

while the dressing functions Eq. (25) of the quark propagator become

$$
A_{p}=\frac{|\mathbf{p}|}{E_{p}}\left(1-\frac{m}{E_{p}} s_{p}\right), \quad B_{p}=\frac{m}{E_{p}}+\frac{\mathbf{p}^{2}}{E_{p}^{2}} s_{p} .
$$

Inserting these expressions into Eq. (40) yields

$$
\begin{aligned}
e_{\mathrm{AD}}= & -4 \int \frac{\mathrm{d}^{3} q}{(2 \pi)^{3}} \frac{E_{q}}{1+w_{q}^{2}} \\
& +g^{2} C_{F} \int \frac{\mathrm{d}^{3} q}{(2 \pi)^{3}} \frac{\mathrm{d}^{3} \ell}{(2 \pi)^{3}} \frac{F(\mathbf{q}-\boldsymbol{\ell})}{E_{q} E_{\ell}} \\
& \times \frac{\left(m+q w_{q}\right)\left(m w_{\ell}-\ell\right)\left(w_{\ell}-\hat{\mathbf{q}} \cdot \hat{\boldsymbol{\ell}}_{w_{q}}\right)}{\left(1+w_{q}^{2}\right)\left(1+w_{\ell}^{2}\right)},
\end{aligned}
$$

where we have introduced the abbreviation

$$
w_{p}=\frac{|\mathbf{p}| s_{p}}{E_{p}} .
$$

Variation of $e_{\mathrm{AD}}$ with respect to $s_{p}$ (or, equivalently, with respect to $w_{p}$ ) yields the gap equation

$$
\begin{aligned}
E_{p} w_{p}= & \frac{g^{2} C_{F}}{2} \int \frac{\mathrm{d}^{3} q}{(2 \pi)^{3}} F(\mathbf{p}-\mathbf{q}) \frac{p}{E_{p}} \frac{q}{E_{q}} \\
& \times \frac{\mathcal{A}(\mathbf{p} ; \mathbf{q})-\hat{\mathbf{p}} \cdot \hat{\mathbf{q}} \mathcal{A}(\mathbf{q} ; \mathbf{p})}{1+w_{q}^{2}},
\end{aligned}
$$


with

$\mathcal{A}(\mathbf{p} ; \mathbf{q})=\left[w_{q}+\frac{m}{2 q}\left(1-w_{q}^{2}\right)\right]\left[1-w_{p}^{2}-2 \frac{m}{p} w_{p}\right]$.

Putting $m=0$ in Eqs. (42) and (43) and approximating the Coulomb potential $F(\mathbf{p})$ [Eq. (30)] by its infrared part $8 \pi \sigma_{\mathrm{C}} / p^{4}$ yields precisely the gap equation obtained by Adler and Davis [25]. Equations (42) and (43) give the extension of their model to finite current quark masses. The integral on the right-hand side of Eq. (42) appears also in Ref. [27], where a slightly extended phenomenological model for the quark-quark interaction was considered.

From the dressing functions [Eq. (41)] we can calculate the quark propagator $Q$ [Eq. (24)]

$$
Q(\mathbf{p})=\frac{\boldsymbol{\alpha} \cdot \hat{\mathbf{p}}\left(p-m w_{p}\right)+\beta\left(m+p w_{p}\right)}{E_{\mathbf{p}}\left(1+w_{p}^{2}\right)},
$$

and after elementary but somewhat lengthy algebra the true quark propagator $S$ [see Eq. (26)] can be cast into the form

$$
S(\mathbf{p})=\frac{\boldsymbol{\alpha} \cdot \mathbf{p}+\beta M_{p}}{2 \sqrt{p^{2}+M_{p}^{2}}}
$$

where the mass function $M_{p}$ is related to the variational kernel $s_{p}$ through

$$
M_{p}=\frac{2 p w_{p}+m\left(1-w_{p}^{2}\right)}{1-w_{p}^{2}-2 \frac{m}{p} w_{p}} .
$$

Equation (44) gives a quasiparticle approximation to the full quark propagator: It has the same form as the free-fermion propagator $S_{0}$ [Eq. (8)] except that the current quark mass $m$ is replaced by a running mass $M_{p}$. Note also that in this case the quark dressing function becomes $Z_{p}=1$.

Equation (45) can be used to trade the kernel $s_{p}$ in the gap equation (42) for the running mass $M_{p}$ yielding

$M(\mathbf{p})=m+\frac{g^{2} C_{F}}{2} \int \frac{\mathrm{d}^{3} q}{(2 \pi)^{3}} \frac{F(\mathbf{p}-\mathbf{q})}{\sqrt{q^{2}+M_{q}^{2}}}\left[M_{q}-\frac{\mathbf{p} \cdot \mathbf{q}}{\mathbf{p}^{2}} M_{p}\right]$.

The same equation has been derived in Ref. [33] from a truncated system of DSEs in the so-called first order formalism.

\section{THE BARE-VERTEX APPROXIMATION}

Let us now return to the full equations of motion of Sec. II with the quark-gluon vertex included. We are interested here mainly in recovering the results of Refs. [10,29] within the present CRDSE approach. For this purpose we replace in the following the full quark-gluon vertex $\bar{\Gamma}$ [Eq. (21)] by the bare one $\bar{\Gamma}_{0}$ [Eq. (13)]. We are aware that this approximation might not yet be entirely sufficient to provide a realistic description of the mechanism of spontaneous breaking of chiral symmetry, i.e. to yield realistic values for quark condensate in agreement with low-energy meson phenomenology. Nevertheless, it is certainly worthwhile to investigate first the bare-vertex approximation in order to get a better understanding of the structure of the equations of motion of the present approach. In addition, the use of a bare quark-gluon vertex is sufficient to carry out the renormalization of these equations, since the leading UV behavior of the dressed vertex agrees with that of the bare one, due to asymptotic freedom.

\section{A. The quark CRDSE}

After replacing the full vertices in the CRDSE (25) by bare ones, the Dirac traces can be worked out and the coupled equations (25) for the dressing functions of the quark propagator reduce in the chiral limit $m=0$ to the set of equations (B1) given in Appendix B. Equations (B1b) and (B1c) for the dressing functions $B_{p}$ and $C_{p}$ can be collected into a single equation for the complex quantity $H=B+\mathrm{i} C$

$$
\begin{aligned}
H_{p}= & s_{p}+\frac{g^{2} C_{F}}{2} \int \frac{\mathrm{d}^{3} q}{(2 \pi)^{3}} \frac{H_{q}^{*}}{\Omega(\mathbf{p}+\mathbf{q}) \Delta_{q}} \\
& \times\left[X_{-}(\mathbf{p}, \mathbf{q}) V(\mathbf{p}, \mathbf{q}) V(\mathbf{q}, \mathbf{p})-X_{+}(\mathbf{p}, \mathbf{q}) W(\mathbf{p}, \mathbf{q}) W(\mathbf{q}, \mathbf{p})\right],
\end{aligned}
$$

where we have introduced the abbreviations

$$
X_{ \pm}(\mathbf{p}, \mathbf{q})=1 \pm \frac{[\hat{\mathbf{p}} \cdot(\mathbf{p}+\mathbf{q})][\hat{\mathbf{q}} \cdot(\mathbf{p}+\mathbf{q})]}{(\mathbf{p}+\mathbf{q})^{2}},
$$

while $\Delta_{q}$ is given by Eq. (33). Similarly, the equations (B1a) and (B1d) for $A_{p}$ and $D_{p}$ can be added and subtracted, yielding

$$
\begin{aligned}
A_{p}+D_{p}= & 1+\frac{g^{2} C_{F}}{2} \int \frac{\mathrm{d}^{3} q}{(2 \pi)^{3}} \frac{A_{q}+D_{q}}{\Omega(\mathbf{p}+\mathbf{q}) \Delta_{q}} \\
& \times\left[X_{-}(\mathbf{p}, \mathbf{q})|V(\mathbf{p}, \mathbf{q})|^{2}+X_{+}(\mathbf{p}, \mathbf{q})|W(\mathbf{p}, \mathbf{q})|^{2}\right], \\
A_{p}-D_{p}= & \frac{g^{2} C_{F}}{2} \int \frac{\mathrm{d}^{3} q}{(2 \pi)^{3}} \frac{A_{q}-D_{q}}{\Omega(\mathbf{p}+\mathbf{q}) \Delta_{q}} \\
& \times\left[X_{-}(\mathbf{p}, \mathbf{q})|V(\mathbf{q}, \mathbf{p})|^{2}+X_{+}(\mathbf{p}, \mathbf{q})|W(\mathbf{q}, \mathbf{p})|^{2}\right] .
\end{aligned}
$$

If the variational vector kernels have the symmetry

$$
|V(\mathbf{p}, \mathbf{q})|=|V(\mathbf{q}, \mathbf{p})|, \quad|W(\mathbf{p}, \mathbf{q})|=|W(\mathbf{q}, \mathbf{p})|
$$

then the Eqs. (B1a) and (B1d) for the form factors $A_{p}$ and $D_{p}$ decouple and there exists always the trivial solution $D_{p}=0$. Finally, notice that for vanishing vector kernels $V=0=W$ these equations reduce to 
$A_{p}=1, \quad B_{p}=\Re s_{p}, \quad C_{p}=\Im s_{p}, \quad D_{p}=0$.

The quark propagator is then entirely determined by the scalar variational kernel $s_{p}$, which corresponds to the BCStype model considered in Refs. [22,25,27], see Sec. IV.

\section{B. Determination of the variational kernels}

From both continuum [33] as well as lattice [34,35] studies there exists no indication that the quark propagator in Coulomb gauge contains a term proportional to the Dirac matrix $\beta \alpha_{i}$ [see Eq. (24)]. Furthermore, when the energy variable of the full propagator is integrated out to yield the equal-time propagator, the term in the quark propagator proportional to the unit matrix vanishes too. Therefore, we expect the physical quark propagator equation (24) to be characterized by $C_{p}=D_{p}=0$. It is not difficult to see that the quark CRDSEs (B1) allow for consistent solutions with
$C_{p}=D_{p}=0$ when the variational kernels $s, V$ and $W$ are real, and the vector kernels $V$ and $W$ are symmetric in the quark momenta. Under these assumptions the quark propagator CRDSEs (B1) reduce to

$$
\begin{aligned}
A_{p}= & 1+g^{2} \frac{C_{F}}{2} \int \frac{\mathrm{d}^{3} q}{(2 \pi)^{3}} \frac{A_{q}}{\Delta_{q} \Omega(\mathbf{p}+\mathbf{q})} \\
& \times\left[X_{-}(\mathbf{p}, \mathbf{q}) V^{2}(\mathbf{p}, \mathbf{q})+X_{+}(\mathbf{p}, \mathbf{q}) W^{2}(\mathbf{p}, \mathbf{q})\right], \\
B_{p}= & s_{p}+g^{2} \frac{C_{F}}{2} \int \frac{\mathrm{d}^{3} q}{(2 \pi)^{3}} \frac{B_{q}}{\Delta_{q} \Omega(\mathbf{p}+\mathbf{q})} \\
& \times\left[X_{-}(\mathbf{p}, \mathbf{q}) V^{2}(\mathbf{p}, \mathbf{q})-X_{+}(\mathbf{p}, \mathbf{q}) W^{2}(\mathbf{p}, \mathbf{q})\right],
\end{aligned}
$$

while the contributions to the energy density [see Eqs. (27), (28), and (32)] become

$$
\begin{aligned}
e_{\mathrm{D}}= & -4 \int \frac{\mathrm{d}^{3} q}{(2 \pi)^{3}} \frac{|\mathbf{q}| A_{q}}{\Delta_{q}} \\
& +2 g^{2} C_{F} \int \frac{\mathrm{d}^{3} q}{(2 \pi)^{3}} \frac{\mathrm{d}^{3} \ell}{(2 \pi)^{3}} \frac{X_{-}(\mathbf{q}, \boldsymbol{\ell}) V(\mathbf{q}, \boldsymbol{\ell})\left(A_{q} A_{\ell}+B_{q} B_{\ell}\right)+X_{+}(\mathbf{q}, \boldsymbol{\ell}) W(\mathbf{q}, \boldsymbol{\ell})\left(A_{q} B_{\ell}+B_{q} A_{\ell}\right)}{\Delta_{q} \Delta_{\ell} \Omega(\mathbf{q}+\boldsymbol{\ell})} \\
e_{E}^{q}= & g^{2} C_{F} \int \frac{\mathrm{d}^{3} q}{(2 \pi)^{3}} \frac{\mathrm{d}^{3} \ell}{(2 \pi)^{3}} \frac{A_{q} A_{\ell}}{\Delta_{q} \Delta_{l}}\left[X_{-}(\mathbf{q}, \boldsymbol{\ell}) V^{2}(\mathbf{q}, \boldsymbol{\ell})+X_{+}(\mathbf{q}, \boldsymbol{\ell}) W^{2}(\mathbf{q}, \boldsymbol{\ell})\right] \\
e_{\mathrm{C}}^{q q}= & -g^{2} \frac{C_{F}}{2} \int \frac{\mathrm{d}^{3} q}{(2 \pi)^{3}} \frac{\mathrm{d}^{3} \ell}{(2 \pi)^{3}} F(\mathbf{q}-\boldsymbol{\ell}) \frac{4 B_{q} B_{\ell}+\hat{\mathbf{q}} \cdot \hat{\boldsymbol{\ell}}\left[A_{q}\left(2-A_{q}\right)-B_{q}^{2}\right]\left[A_{\ell}\left(2-A_{\ell}\right)-B_{\ell}^{2}\right]}{\Delta_{q} \Delta_{\ell}}
\end{aligned}
$$

with $\Delta$ [Eq. (33)] reducing to

$$
\Delta_{p}=A_{p}^{2}+B_{p}^{2}
$$

The energy density contributions (49) contain the scalar kernel $s_{p}$ only implicitly through the dressing functions $A_{p}$ and $B_{p}$, while the vector kernels $V$ and $W$ enter both explicitly and implicitly. From Eq. (48a) we find the derivatives of the dressing function $A_{p}$ with respect to the vector kernels

$$
\begin{aligned}
& \frac{\delta A_{k}}{\delta V(\mathbf{p}, \mathbf{q})}=g^{2} \frac{C_{F}}{2} \frac{X_{-}(\mathbf{p}, \mathbf{q})}{\Omega(\mathbf{p}+\mathbf{q})} V(\mathbf{p}, \mathbf{q})\left[(2 \pi)^{3} \delta(\mathbf{k}-\mathbf{p}) \frac{A_{q}}{\Delta_{q}}+(2 \pi)^{3} \delta(\mathbf{k}-\mathbf{q}) \frac{A_{p}}{\Delta_{p}}\right]+\cdots \\
& \frac{\delta A_{k}}{\delta W(\mathbf{p}, \mathbf{q})}=g^{2} \frac{C_{F}}{2} \frac{X_{+}(\mathbf{p}, \mathbf{q})}{\Omega(\mathbf{p}+\mathbf{q})} W(\mathbf{p}, \mathbf{q})\left[(2 \pi)^{3} \delta(\mathbf{k}-\mathbf{p}) \frac{A_{q}}{\Delta_{q}}+(2 \pi)^{3} \delta(\mathbf{k}-\mathbf{q}) \frac{A_{p}}{\Delta_{p}}\right]+\cdots
\end{aligned}
$$

and similarly the derivatives of $B_{p}$

$$
\begin{gathered}
\frac{\delta B_{k}}{\delta V(\mathbf{p}, \mathbf{q})}=g^{2} \frac{C_{F}}{2} \frac{X_{-}(\mathbf{p}, \mathbf{q})}{\Omega(\mathbf{p}+\mathbf{q})} V(\mathbf{p}, \mathbf{q})\left[(2 \pi)^{3} \delta(\mathbf{k}-\mathbf{p}) \frac{B_{q}}{\Delta_{q}}+(2 \pi)^{3} \delta(\mathbf{k}-\mathbf{q}) \frac{B_{p}}{\Delta_{p}}\right]+\cdots \\
\frac{\delta B_{k}}{\delta W(\mathbf{p}, \mathbf{q})}=-g^{2} \frac{C_{F}}{2} \frac{X_{+}(\mathbf{p}, \mathbf{q})}{\Omega(\mathbf{p}+\mathbf{q})} W(\mathbf{p}, \mathbf{q})\left[(2 \pi)^{3} \delta(\mathbf{k}-\mathbf{p}) \frac{B_{q}}{\Delta_{q}}+(2 \pi)^{3} \delta(\mathbf{k}-\mathbf{q}) \frac{B_{p}}{\Delta_{p}}\right]+\cdots
\end{gathered}
$$


The ellipsis on the right-hand side of these equations stand for the one-loop terms, which we will usually neglect since they would give rise to more than one loop in the equations of motion of the vector kernels.

In the same way we can evaluate the functional derivatives of the dressing functions $A_{p}$ and $B_{p}$ with respect to the scalar kernel $s_{p}$

$$
\begin{gathered}
\frac{\delta A_{k}}{\delta s_{p}}=-g^{2} \frac{C_{F}}{2} \int \frac{\mathrm{d}^{3} q}{(2 \pi)^{3}} \frac{X_{-}(\mathbf{k}, \mathbf{q}) V^{2}(\mathbf{k}, \mathbf{q})+X_{+}(\mathbf{k}, \mathbf{q}) W^{2}(\mathbf{k}, \mathbf{q})}{\Delta_{q}^{2} \Omega(\mathbf{k}+\mathbf{q})}\left[\left(A_{q}^{2}-B_{q}^{2}\right) \frac{\delta A_{q}}{\delta s_{p}}+2 A_{q} B_{q} \frac{\delta B_{q}}{\delta s_{p}}\right], \\
\frac{\delta B_{k}}{\delta s_{p}}=(2 \pi)^{3} \delta(\mathbf{p}-\mathbf{k})+g^{2} \frac{C_{F}}{2} \int \frac{\mathrm{d}^{3} q}{(2 \pi)^{3}} \frac{X_{-}(\mathbf{k}, \mathbf{q}) V^{2}(\mathbf{k}, \mathbf{q})-X_{+}(\mathbf{k}, \mathbf{q}) W^{2}(\mathbf{k}, \mathbf{q})}{\Delta_{q}^{2} \Omega(\mathbf{k}+\mathbf{q})}\left[\left(A_{q}^{2}-B_{q}^{2}\right) \frac{\delta B_{q}}{\delta s_{p}}-2 A_{q} B_{q} \frac{\delta A_{q}}{\delta s_{p}}\right] .
\end{gathered}
$$

At one-loop order the previous equations reduce to

$$
\begin{aligned}
& \frac{\delta A_{k}}{\delta s_{p}}=-g^{2} C_{F} \frac{A_{p} B_{p}}{\Delta_{p}^{2} \Omega(\mathbf{k}+\mathbf{p})}\left[X_{-}(\mathbf{k}, \mathbf{p}) V^{2}(\mathbf{k}, \mathbf{p})+X_{+}(\mathbf{k}, \mathbf{p}) W^{2}(\mathbf{k}, \mathbf{p})\right]+\cdots \\
& \frac{\delta B_{k}}{\delta s_{p}}=(2 \pi)^{3} \delta(\mathbf{p}-\mathbf{k})+g^{2} \frac{C_{F}}{2} \frac{A_{p}^{2}-B_{p}^{2}}{\Delta_{p}^{2} \Omega(\mathbf{k}+\mathbf{p})}\left[X_{-}(\mathbf{k}, \mathbf{p}) V^{2}(\mathbf{k}, \mathbf{p})-X_{+}(\mathbf{k}, \mathbf{p}) W^{2}(\mathbf{k}, \mathbf{p})\right]+\cdots
\end{aligned}
$$

In a diagrammatic language, differentiating with respect to the vector kernel implies removing one quark-gluon vertex from the diagram. Since the energy contributions contain at most two loops, the variational equations for $V$ and $W$ are free of loops. To this order, we can ignore the Coulomb energy equation (49c) and include only the explicit dependence on $V$ and $W$ in the second term of Eq. (49a) and in Eq. (49b), yielding

$$
\begin{aligned}
\frac{\delta\left(e_{\mathrm{D}}^{(1)}+e_{E}^{q}\right)}{\delta V(\mathbf{p}, \mathbf{q})}= & 2 g^{2} C_{F} \frac{X_{-}(\mathbf{p}, \mathbf{q})}{\Delta_{p} \Delta_{q}} \\
& \times\left[\frac{A_{p} A_{q}+B_{p} B_{q}}{\Omega(\mathbf{p}+\mathbf{q})}+A_{p} A_{q} V(\mathbf{p}, \mathbf{q})\right]
\end{aligned}
$$

as well as

$$
\begin{aligned}
\frac{\delta\left(e_{\mathrm{D}}^{(1)}+e_{E}^{q}\right)}{\delta W(\mathbf{p}, \mathbf{q})}= & 2 g^{2} C_{F} \frac{X_{+}(\mathbf{p}, \mathbf{q})}{\Delta_{p} \Delta_{q}} \\
& \times\left[\frac{A_{p} B_{q}+B_{p} A_{q}}{\Omega(\mathbf{p}+\mathbf{q})}+A_{p} A_{q} W(\mathbf{p}, \mathbf{q})\right] .
\end{aligned}
$$

In the first term of Eq. (49a), however, we must take into account also the dependence of the dressing functions $A_{p}$ and $B_{p}$ on the kernels $V$ and $W$. This yields

$$
\begin{aligned}
\frac{\delta e_{\mathrm{D}}^{(0)}}{\delta V(\mathbf{p}, \mathbf{q})}= & 4 \int \frac{\mathrm{d}^{3} \ell}{(2 \pi)^{3}} \frac{|\boldsymbol{\ell}|}{\Delta_{\ell}^{2}} \\
& \times\left\{\left(A_{\ell}^{2}-B_{\ell}^{2}\right) \frac{\delta A_{\ell}}{\delta V(\mathbf{p}, \mathbf{q})}+2 A_{\ell} B_{\ell} \frac{\delta B_{\ell}}{\delta V(\mathbf{p}, \mathbf{q})}\right\}
\end{aligned}
$$

and by using Eqs. (51) and (53) we find

$$
\begin{aligned}
\frac{\delta e_{\mathrm{D}}^{(0)}}{\delta V(\mathbf{p}, \mathbf{q})}= & 2 g^{2} C_{F} \frac{X_{-}(\mathbf{p}, \mathbf{q})}{\Omega(\mathbf{p}+\mathbf{q})} V(\mathbf{p}, \mathbf{q}) \\
& \times\left\{\frac{|\mathbf{p}|}{\Delta_{p}^{2}}\left[\left(A_{p}^{2}-B_{p}^{2}\right) \frac{A_{q}}{\Delta_{q}}+2 A_{p} B_{p} \frac{B_{q}}{\Delta_{q}}\right]\right. \\
& \left.+\frac{|\mathbf{q}|}{\Delta_{q}^{2}}\left[\left(A_{q}^{2}-B_{q}^{2}\right) \frac{A_{p}}{\Delta_{p}}+2 A_{q} B_{q} \frac{B_{p}}{\Delta_{p}}\right]\right\} .
\end{aligned}
$$

Requiring that the sum of Eqs. (56) and (57) vanishes fixes the vector kernel $V$ to

$$
V(\mathbf{p}, \mathbf{q})=-\frac{A_{p} A_{q}+B_{p} B_{q}}{A_{p} A_{q} \Omega(\mathbf{p}+\mathbf{q})+|\mathbf{p}| \frac{A_{q}\left(A_{p}^{2}-B_{p}^{2}\right)+2 A_{p} B_{p} B_{q}}{\Delta_{p}}+|\mathbf{q}| \frac{A_{p}\left(A_{q}^{2}-B_{q}^{2}\right)+2 A_{q} B_{p} B_{q}}{\Delta_{p}}} .
$$

To simplify this and the following expressions we introduce the ratio

$$
b_{p} \equiv \frac{B_{p}}{A_{p}}
$$

$$
V(\mathbf{p}, \mathbf{q})=-\frac{1+b_{p} b_{q}}{\Omega(\mathbf{p}+\mathbf{q})+\frac{|\mathbf{p}|}{A_{p}} \frac{1-b_{p}^{2}+2 b_{p} b_{q}}{1+b_{p}^{2}}+\frac{|\mathbf{q}|}{A_{q}} \frac{1-b_{q}^{2}+2 b_{p} b_{q}}{1+b_{q}^{2}}} .
$$


At leading order we find from Eq. (48) $A_{p}=1$ and $b_{p}=s_{p}$, and Eq. (60) reduces to the kernel found in Ref. [10]. Furthermore, at large momenta we recover the leading-order perturbative result [36].

The variation of the energy with respect to $W$ is carried out in an analogous way by using Eqs. (52) and (54). This yields the equation of motion

$$
W(\mathbf{p}, \mathbf{q})=-\frac{b_{p}+b_{q}}{\Omega(\mathbf{p}+\mathbf{q})+\frac{|\mathbf{p}|}{A_{p}} \frac{1-b_{p}^{2}-2 b_{p} b_{q}}{1+b_{p}^{2}}+\frac{|\mathbf{q}|}{A_{q}} \frac{1-b_{q}^{2}-2 b_{p} b_{q}}{1+b_{q}^{2}}} .
$$

Also this kernel reduces to the one found in Ref. [10] at leading order. Both kernels $V$ and $W$ turn out to be real and negative, as we might have expected from $e_{\mathrm{D}}^{(1)}[\mathrm{Eq} .(\mathrm{C} 1)]$ : this is the only energy contribution involving the variational vector kernels linearly. This energy contribution vanishes if the quark-gluon coupling is neglected in the vacuum wave functional, i.e. for $V=0=W$. Negative vector kernels $V$ and $W$ are energetically favored since they make $e_{\mathrm{D}}^{(1)}$ negative.

The variation of the energy density with respect to the scalar kernel $s_{p}$ is slightly more involved than the variational derivative with respect to the vector kernels. For the second term in the single-particle energy density equation (49a), as well as for the contributions of the gluonic kinetic term equation (49b) and of the Coulomb interaction equation $(49 \mathrm{c})$ it is sufficient to keep only the leading order of Eq. (55), while for the first term in Eq. (49a) we need also the one-loop contributions. Then the variation with respect to $s_{p}$ yields

$$
\begin{aligned}
\frac{b_{p}|\mathbf{p}|}{A_{p}^{2}\left(1+b_{p}^{2}\right)^{2}}= & \frac{g^{2} C_{F}}{2 A_{p}^{2}\left(1+b_{p}^{2}\right)^{2}} \int \frac{\mathrm{d}^{3} q}{(2 \pi)^{3}} \frac{1}{A_{q}\left(1+b_{q}^{2}\right)}\left\{b_{p}\left[X_{-}(\mathbf{p}, \mathbf{q}) V^{2}(\mathbf{p}, \mathbf{q})+X_{+}(\mathbf{p}, \mathbf{q}) W^{2}(\mathbf{p}, \mathbf{q})\right]\right. \\
& -\frac{|\mathbf{q}|}{A_{q}\left(1+b_{q}^{2}\right) \Omega(\mathbf{p}+\mathbf{q})}\left[X_{-}(\mathbf{p}, \mathbf{q}) V^{2}(\mathbf{p}, \mathbf{q})\left[\left(1-b_{p}^{2}\right) b_{q}-b_{p}\left(1-b_{q}^{2}\right)\right]\right. \\
& \left.-X_{+}(\mathbf{p}, \mathbf{q}) W^{2}(\mathbf{p}, \mathbf{q})\left[\left(1-b_{p}^{2}\right) b_{q}+b_{p}\left(1-b_{q}^{2}\right)\right]\right] \\
& \left.-\frac{1}{\Omega(\mathbf{p}+\mathbf{q})}\left[X_{-}(\mathbf{p}, \mathbf{q}) V(\mathbf{p}, \mathbf{q})\left[\left(1-b_{p}^{2}\right) b_{q}-2 b_{p}\right)\right]+X_{+}(\mathbf{p}, \mathbf{q}) W(\mathbf{p}, \mathbf{q})\left[1-b_{p}^{2}-2 b_{p} b_{q}\right]\right] \\
& \left.+F(\mathbf{p}-\mathbf{q})\left[b_{q}\left(1-b_{p}^{2}\right)-\hat{\mathbf{p}} \cdot \hat{\mathbf{q}}\left(2-A_{q}\left(1+b_{p}^{2}\right)\right)\right]\right\},
\end{aligned}
$$

where we have expressed the resulting equations in terms of $b_{p}$ [Eq. (59)] instead of $s_{p}$. In order to reproduce the loop expansion of Ref. [10] $]^{2}$ on the right-hand side of Eq. (62) it is sufficient to replace $b_{p} \rightarrow s_{p}$ and $A_{p} \rightarrow 1$, while on the lefthand side the factor

$$
\frac{b_{p}}{A_{p}^{2}\left(1+b_{p}^{2}\right)^{2}}=\frac{A_{p} B_{p}}{\left(A_{p}^{2}+B_{p}^{2}\right)^{2}}
$$

has to be expanded up to one-loop order by means of Eq. (48), yielding

$$
\begin{aligned}
\frac{b_{p}}{A_{p}^{2}\left(1+b_{p}^{2}\right)^{2}}= & \frac{s_{p}}{\left(1+s_{p}^{2}\right)^{2}}+\frac{1}{\left(1+s_{p}^{2}\right)^{3}} \frac{g^{2} C_{F}}{2} \int \frac{\mathrm{d}^{3} q}{(2 \pi)^{3}} \frac{1}{\left(1+s_{q}^{2}\right) \Omega(\mathbf{p}+\mathbf{q})} \\
& \times\left\{X_{-}(\mathbf{p}, \mathbf{q}) V^{2}(\mathbf{p}, \mathbf{q})\left[s_{p}\left(s_{p}^{2}-3\right)+s_{q}\left(1-3 s_{p}^{2}\right)\right]+X_{+}(\mathbf{p}, \mathbf{q}) W^{2}(\mathbf{p}, \mathbf{q})\left[s_{p}\left(s_{p}^{2}-3\right)-s_{q}\left(1-3 s_{p}^{2}\right)\right]\right\} .
\end{aligned}
$$

With these replacements Eq. (62) reduces precisely to the gap equation found in Refs. [10,29], which is explicitly given in our notation in Appendix D. In fact, the same result may be obtained by expanding the dressing functions (48) at one-loop order in the energy density contributions (49) and taking the variation afterwards.

\footnotetext{
${ }^{2}$ The present approach allows one to go beyond this loop expansion.
}

\section{RENORMALIZED QUARK PROPAGATOR AND CHIRAL CONDENSATE}

The gap equation (D1) has been solved numerically for the variational kernel $s_{p}$ in Ref. [29]. The renormalization of the quark propagator equation (34) was ignored and the quark condensate was evaluated from the leading-order (in the number of quark loops) propagator

$$
S(\mathbf{p})=\frac{\left(1-s_{p}^{2}\right) \boldsymbol{\alpha} \cdot \hat{\mathbf{p}}+2 s_{p} \beta}{2\left(1+s_{p}^{2}\right)}
$$


which arises from the full propagator equation (34) by putting $A_{p}=1$ and $B_{p}=s_{p}$, which are the zero-loop expressions [see Eq. (47)]. The coupling $g$ was then chosen to reproduce the phenomenological value of the quark condensate

$$
\langle\bar{q} q\rangle=-\int \frac{\mathrm{d}^{3} p}{(2 \pi)^{3}} \operatorname{tr}[\beta S(\mathbf{p})]=-\frac{2 N_{c}}{\pi^{2}} \int \mathrm{d} p p^{2} \frac{s_{p}}{1+s_{p}^{2}} .
$$

Here we go beyond Ref. [29] and consistently calculate the quark propagator up to including one-loop order. This should be sufficient to investigate the renormalization properties of the quark propagator.

To one-loop order we can replace the denominator $\Delta_{p}$ [Eq. (50)] in Eq. (48) by its leading-order expression $A_{p}=1, B_{p}=s_{p}$. Then Eq. (48) becomes

$$
\begin{aligned}
& A_{p}=1+g^{2} \frac{C_{F}}{2} \int \frac{\mathrm{d}^{3} q}{(2 \pi)^{3}} \frac{X_{-}(\mathbf{p}, \mathbf{q}) V^{2}(\mathbf{p}, \mathbf{q})+X_{+}(\mathbf{p}, \mathbf{q}) W^{2}(\mathbf{p}, \mathbf{q})}{\left(1+s_{q}^{2}\right) \Omega(\mathbf{p}+\mathbf{q})} \equiv 1+I_{A}(p, \Lambda), \\
& B_{p}=s_{p}+g^{2} \frac{C_{F}}{2} \int \frac{\mathrm{d}^{3} q}{(2 \pi)^{3}} s_{q} \frac{X_{-}(\mathbf{p}, \mathbf{q}) V^{2}(\mathbf{p}, \mathbf{q})-X_{+}(\mathbf{p}, \mathbf{q}) W^{2}(\mathbf{p}, \mathbf{q})}{\left(1+s_{q}^{2}\right) \Omega(\mathbf{p}+\mathbf{q})} \equiv s_{p}+I_{B}(p, \Lambda),
\end{aligned}
$$

where $V$ and $W$ are given by Eqs. (60) and (61) with $b_{p}$ replaced by $s_{p}$ and $A_{p}$ replaced by 1 ; furthermore, $\Lambda$ is a momentum cutoff. A quick calculation shows that the loop integral $I_{B}$ is convergent while $I_{A}$ is logarithmically divergent

$$
I_{A}(p, \Lambda)=\frac{g^{2} C_{F}}{(4 \pi)^{2}}\left(1+s_{p}^{2}\right) \ln \Lambda+\text { finite terms. }
$$

At first sight, the appearance of a momentum-dependent divergence seems to spoil multiplicative renormalizability. However, this is not the case, as we will show now. Expanding the quark propagator Eq. (34) at one-loop order we obtain

$$
S(\mathbf{p}, \Lambda)=\frac{1}{1+s_{p}^{2}}\left\{\frac{\boldsymbol{\alpha} \cdot \hat{\mathbf{p}}}{2}\left[\left(1-s_{p}^{2}\right)\left(1-\frac{2 I_{A}(p, \Lambda)}{1+s_{p}^{2}}\right)-\frac{4 s_{p} I_{B}(p, \Lambda)}{1+s_{p}^{2}}\right]+\beta\left[s_{p}\left(1-\frac{2 I_{A}(p, \Lambda)}{1+s_{p}^{2}}\right)+\frac{1-s_{p}^{2}}{1+s_{p}^{2}} I_{B}(p, \Lambda)\right]\right\} .
$$

Inserting here Eq. (64) one finds that the momentumdependent part of the logarithmic divergence cancels. The remaining part of the UV divergence can be removed by the perturbative one-loop renormalization constant, which in the $\overline{\mathrm{MS}}$ scheme reads $[36,37]$

$Z_{2}(\Lambda, \mu)=1-\frac{g^{2} C_{F}}{(4 \pi)^{2}}\left[\ln \frac{\Lambda^{2}}{\mu^{2}}+\ln 4 \pi-\gamma_{\mathrm{E}}\right] \equiv 1-\delta z_{2}(\Lambda, \mu)$

With this expression we can define a renormalized propagator

$$
S(\mathbf{p}, \mu)=f_{\alpha}(p, \mu) \boldsymbol{\alpha} \cdot \hat{\mathbf{p}}+f_{\beta}(p, \mu) \beta
$$

where

$$
\begin{aligned}
f_{\alpha}(p, \mu)= & \frac{1}{2} \frac{1-s_{p}^{2}}{1+s_{p}^{2}}\left[1-\frac{2 I_{A}(p, \Lambda)}{1+s_{p}^{2}}+\delta z_{2}(\Lambda, \mu)\right] \\
& -\frac{2 s_{p}}{\left(1+s_{p}^{2}\right)^{2}} I_{B}(p), \\
f_{\beta}(p, \mu)= & \frac{s_{p}}{1+s_{p}^{2}}\left[1-\frac{2 I_{A}(p, \Lambda)}{1+s_{p}^{2}}+\delta z_{2}(\Lambda, \mu)\right] \\
& +\frac{1-s_{p}^{2}}{\left(1+s_{p}^{2}\right)^{2}} I_{B}(p) .
\end{aligned}
$$

By means of Eqs. (64) and (65) one finds that Eq. (67) is indeed finite when the cutoff $\Lambda$ is removed.

It is important to note that without the vector kernel $W$, i.e. without the Dirac structure $\beta \alpha_{i}$ in the bare quark-gluon vertex of the quark wave functional, the term $1+s_{p}^{2}$ in Eq. (64) would reduce to 1 and the physical quark propagator would no longer be multiplicatively renormalizable [cf. Eq. (67)]. Although the vector kernel $W$ [Eq. (61)] is purely nonperturbative in nature, its presence in the quark wave functional [Eqs. (9), (10), (17)] is necessary to ensure multiplicative renormalizability of the propagator.

The renormalization point dependent quark condensate in the $\overline{\mathrm{MS}}$ scheme is usually quoted at the renormalization scale $\mu=2 \mathrm{GeV}$. At this scale the running strong coupling constant has the value $\alpha_{s}(2 \mathrm{GeV})=0.30(1)$ [38]. Solving the gap equation (D1) with this value of $\alpha_{s}$ yields the dressing functions [Eq. (67)] shown in Fig. 2. The resulting chiral condensate is

$$
\langle\bar{q} q\rangle \simeq\left(-0.31 \sqrt{\sigma_{\mathrm{C}}}\right)^{3} .
$$

The scale in our calculations is fixed by the Coulomb string tension $\sigma_{\mathrm{C}}$ occurring in the color Coulomb potential Eq. (30). Lattice and continuum calculations [39-41] quote values of the Coulomb string tension from 2.5 to as large as 

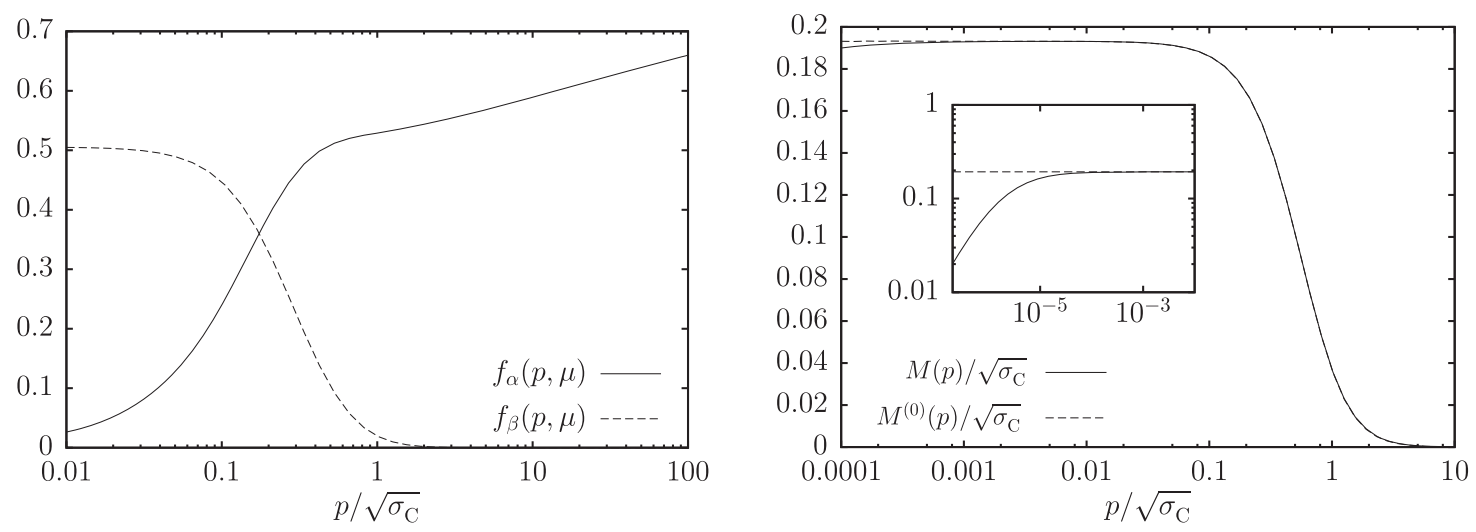

FIG. 2. (left) Dressing functions $f_{\alpha}$ and $f_{\beta}$ of the renormalized quark propagator. (right) Mass function of the renormalized [Eq. (69), continuous line] and unrenormalized [Eq. (70), dashed line] quark propagator.

4 times the Wilson string tension $\sigma=(440 \mathrm{MeV})^{2}$, which gives us $\sqrt{\sigma_{\mathrm{C}}}$ in the range from 696 to $880 \mathrm{MeV}$. This yields a (renormalization point dependent) chiral condensate in the range between $(-216 \mathrm{MeV})^{3}$ and $(-270 \mathrm{MeV})^{3}$. Lattice simulations and chiral perturbation theory calculations yield for the chiral condensate values in a similar range $[1,2,42-44]$.

The renormalized quark propagator Eq. (66) can be cast into the form Eq. (35)

$$
S(\mathbf{p}, \mu)=Z_{p} \frac{\boldsymbol{\alpha} \cdot \mathbf{p}+\beta M_{p}}{2 \sqrt{\mathbf{p}^{2}+M_{p}^{2}}}
$$

where the mass function $M_{p}$ and the dressing function $Z_{p}$ are related to $f_{\alpha}$ and $f_{\beta}$ [Eq. (67)] by

$M_{p}=\frac{|\mathbf{p}| f_{\beta}(p, \mu)}{f_{\alpha}(p, \mu)}, \quad Z_{p}=2 \sqrt{f_{\alpha}^{2}(p, \mu)+f_{\beta}^{2}(p, \mu)}$.

From the definition of the mass function [Eq. (69)] it is clear that if $f_{\alpha}$ does not vanish for $\mathbf{p}=0$ the mass function is bound to vanish in the deep infrared. Our numerical results show that while $s(0)=1, I_{B}(0)$ is very small but not vanishing. The reason for this behavior is the fact that the denominators of the vector kernels $V$ [Eq. (60)] and $W$ [Eq. (61)] are not the same. We believe that this is an artifact of the one-loop expansion. The mass function equation (69) stays however constant over almost 3 orders of magnitude before slowly bending over (see Fig. 2). Furthermore, the integral $I_{B}(p)$ is rather small in comparison to the (renormalized) integral $I_{A}$. While the latter has an important effect on the chiral condensate, the mass function equation (69) is, apart from the deep IR, almost indistinguishable from the mass function of Ref. [29] extracted from the unrenormalized quark propagator equation (63)

$$
M^{(0)}(p)=\frac{2 p s_{p}}{1-s_{p}^{2}}
$$

as shown in Fig. 2. While our mass function vanishes in the deep infrared, the plateau value reads

$$
M_{\mathrm{IR}} \simeq 0.19 \sqrt{\sigma_{\mathrm{C}}},
$$

which, due to the uncertainty in the Coulomb string tension, is in the range between 135 and $170 \mathrm{MeV}$.

\section{MASS FUNCTION IN THE FULL AND STATIC PROPAGATOR}

As mentioned before, in Ref. [29] the renormalization of the propagator was ignored and the value of the quarkgluon coupling constant was chosen to reproduce the phenomenological value of the chiral condensate. The mass function, however, was not significantly enhanced in comparison to the Adler-Davis model [25] (see Sec. IV), showing an infrared value of $135 \mathrm{MeV}$ (for $\sigma_{\mathrm{C}}=2.5 \sigma$ ). Similar results have been obtained also in the previous section: although our rough one-loop calculation is capable of reproducing the correct value of the chiral condensate, the mass function is not significantly influenced by the coupling to the transverse gluons. This seems at odds with the common lore that the infrared value of the mass function should be around the value of the constituent quark mass, i.e. roughly $300 \mathrm{MeV}$. Here we show that this apparent contradiction might result from comparing the mass functions of the full and equal-time propagators. Before discussing this issue in Coulomb gauge we address the question in Landau gauge, for which we have solutions of the Dyson-Schwinger equations at our disposal. ${ }^{3}$

Suppressing color indices, the quark propagator in Landau gauge is usually written as

\footnotetext{
${ }^{3} \mathrm{We}$ are aware of the fact that the quark propagator itself and hence also the extracted effective quark mass is gauge dependent. Here we show that besides the gauge dependence the effective mass extracted from a static (time-independent) propagator differs drastically from the mass extracted from the full (time-dependent) propagator.
} 


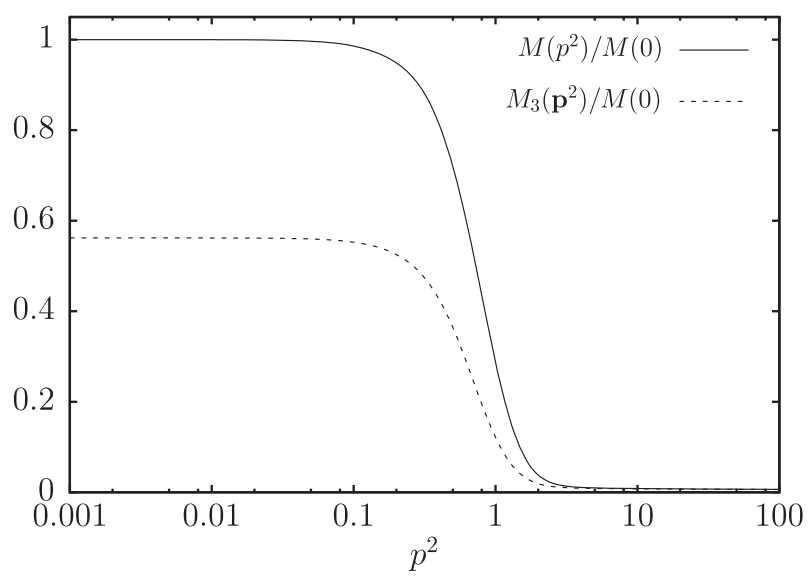

(a)

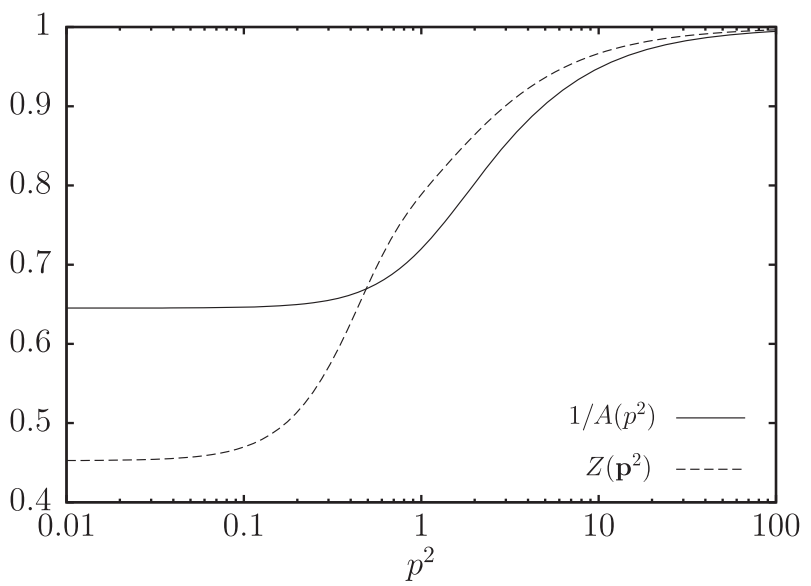

(b)

FIG. 3. (a) Comparison between the full mass function $M\left(p^{2}\right)$ in Landau gauge (continuous line) and the mass function $M_{3}\left(\mathbf{p}^{2}\right)$ of the equal-time propagator (dashed line). (b) Dressing function of the full (continuous line) and equal-time propagator (dashed line).

$$
S(p)=\frac{1}{-\mathrm{i} \not p A\left(p^{2}\right)+B\left(p^{2}\right)}=\frac{1}{A\left(p^{2}\right)} \frac{\mathrm{i} \not p+M\left(p^{2}\right)}{p^{2}+M^{2}\left(p^{2}\right)},
$$

where the quark mass function $M$ is defined as $M\left(p^{2}\right)=B\left(p^{2}\right) / A\left(p^{2}\right)$. At tree level we have $A=1$ and $B=M=m$, with $m$ being the bare current quark mass. The equal-time propagator $S_{3}(\mathbf{p})$ is obtained from the full one $S(p)$ by integrating out the energy component $p_{4}$ of the four-momentum

$$
S_{3}(\mathbf{p})=\int \frac{\mathrm{d} p_{4}}{2 \pi} S(p)
$$

For symmetry reasons the contribution proportional to $\gamma_{4} p_{4}$ vanishes and we are left with

$$
\begin{aligned}
S_{3}(\mathbf{p})= & \mathrm{i} \gamma \cdot \mathbf{p} \int \frac{\mathrm{d} p_{4}}{2 \pi} \frac{1}{A\left(p_{4}^{2}+\mathbf{p}^{2}\right)} \frac{1}{p_{4}^{2}+\mathbf{p}^{2}+M^{2}\left(p_{4}^{2}+\mathbf{p}^{2}\right)} \\
& +\int \frac{\mathrm{d} p_{4}}{2 \pi} \frac{1}{A\left(p_{4}^{2}+\mathbf{p}^{2}\right)} \frac{M\left(p_{4}^{2}+\mathbf{p}^{2}\right)}{p_{4}^{2}+\mathbf{p}^{2}+M^{2}\left(p_{4}^{2}+\mathbf{p}^{2}\right)} .
\end{aligned}
$$

Analogously to the definition of the quark mass function $M$ we can introduce the equal-time mass function $M_{3}\left(\mathbf{p}^{2}\right)$ as ratio of the coefficients of the $\mathbb{1}$ and $\gamma^{i}$ terms of the equaltime propagator, yielding

$$
M_{3}\left(\mathbf{p}^{2}\right)=\frac{\int_{0}^{\infty} \mathrm{d} p_{4} \frac{1}{A\left(p_{4}^{2}+\mathbf{p}^{2}\right)} \frac{M\left(p_{4}^{2}+\mathbf{p}^{2}\right)}{p_{4}^{2}+\mathbf{p}^{2}+M^{2}\left(p_{4}^{2}+\mathbf{p}^{2}\right)}}{\int_{0}^{\infty} \mathrm{d} p_{4} \frac{1}{A\left(p_{4}^{2}+\mathbf{p}^{2}\right)} \frac{1}{p_{4}^{2}+\mathbf{p}^{2}+M^{2}\left(p_{4}^{2}+\mathbf{p}^{2}\right)}} .
$$

Numerical solutions for the mass function always show a monotonically decreasing function of the four-momentum.
Therefore, since $M\left(p^{2}\right) \leq M(0)$ we see from Eq. (72) that $M_{3}(0)<M(0)$. For typical results for the Landau gauge quark propagator we find that $M_{3}(0)$ lies between $50 \%$ and $60 \%$ of $M(0)$, see Fig. 3(a). Furthermore, the equal-time quark propagator Eq. (71) can be brought into the form (68)

$$
S_{3}(\mathbf{p})=Z\left(\mathbf{p}^{2}\right) \frac{\mathrm{i} \gamma \cdot \mathbf{p}+M_{3}\left(\mathbf{p}^{2}\right)}{2 \sqrt{\mathbf{p}^{2}+M_{3}^{2}\left(\mathbf{p}^{2}\right)}} .
$$

Figure 3(b) shows both $Z$ and $A^{-1}$.

The situation might be similar in Coulomb gauge. Being noncovariant, the propagator depends separately on $p_{4}$ and $\mathbf{p}$ and has therefore four Dirac components instead of two

$$
\begin{aligned}
S^{-1}(p)= & -\mathrm{i} \gamma_{4} p_{4} A_{t}\left(p_{4}, \mathbf{p}\right)-\mathrm{i} \gamma \cdot \mathbf{p} A_{s}\left(p_{4}, \mathbf{p}\right) \\
& -\mathrm{i} \gamma_{4} p_{4} \gamma \cdot \mathbf{p} A_{d}\left(p_{4}, \mathbf{p}\right)+B\left(p_{4}, \mathbf{p}\right) .
\end{aligned}
$$

The mixed structure $\gamma_{4} \gamma_{i}$ does not arise at one-loop level in perturbation theory [37] and is not found in lattice calculations [34,35] either; therefore we will set $A_{d}=0$ in the following. The propagator in Coulomb gauge takes therefore the form

$$
S(p)=\frac{\mathrm{i} \gamma_{4} p_{4} A_{t}\left(p_{4}, \mathbf{p}\right)+\mathrm{i} \gamma \cdot \mathbf{p} A_{s}\left(p_{4}, \mathbf{p}\right)+B\left(p_{4}, \mathbf{p}\right)}{p_{4}^{2} A_{t}^{2}\left(p_{4}, \mathbf{p}\right)+\mathbf{p}^{2} A_{s}^{2}\left(p_{4}, \mathbf{p}\right)+B^{2}\left(p_{4}, \mathbf{p}\right)} .
$$

Analogously to Eq. (72) the equal-time mass function in Coulomb gauge is given by

$$
M_{3}(\mathbf{p})=\frac{\int_{0}^{\infty} \mathrm{d} p_{4} \frac{B\left(p_{4}, \mathbf{p}\right)}{p_{4}^{2} A_{t}^{2}\left(p_{4}, \mathbf{p}\right)+\mathbf{p}^{2} A_{s}^{2}\left(p_{4}, \mathbf{p}\right)+B^{2}\left(p_{4}, \mathbf{p}\right)}}{\int_{0}^{\infty} \mathrm{d} p_{4} \frac{A_{s}\left(p_{4}, \mathbf{p}\right)}{p_{4}^{2} A_{t}^{2}\left(p_{4}, \mathbf{p}\right)+\mathbf{p}^{2} A_{s}^{2}\left(p_{4}, \mathbf{p}\right)+B^{2}\left(p_{4}, \mathbf{p}\right)}} .
$$


As for the quark propagator in Landau gauge we expect also in Coulomb gauge that the effective quark mass extracted from the static propagator is considerably smaller than the one extracted from the four-dimensional propagator.

\section{CONCLUSIONS}

The gap equation of Ref. [29] has been rederived within the framework of the canonical recursive Dyson-Schwinger equations. We have shown that the additional Dirac structure in the bare quark-gluon vertex of the vacuum wave functional not only eliminates the UV divergences from the gap equation (as shown already in Refs. [10,29]) but is also crucial to ensure multiplicative renormalizability of the quark propagator. We have performed a quenched semiperturbative calculation assuming a bare quark-gluon vertex. Unlike the covariant functional approaches in Landau gauge, where the dressing of the (four-dimensional) quark-gluon vertex is crucial for obtaining spontaneous breaking of chiral symmetry, in the present Hamiltonian approach the bare quark-gluon vertex in the vacuum wave functional is sufficient to reproduce the phenomenological value of the quark condensate. In the present approach the dominant IR contribution, which triggers the spontaneous breaking of chiral symmetry, comes from the confining Coulomb potential. We have also shown that, depending on the details of the momentum dependence, the effective quark mass obtained in the Hamiltonian approach cannot be compared with the (constituent) mass extracted from the corresponding fourdimensional propagator and is expected to be considerably smaller than the latter. The results obtained in the present paper are quite encouraging for a fully self-consistent solution of the coupled variational and CRDSEs. In a first step towards this goal we will solve the CRDSE for the quark-gluon vertex.

\section{ACKNOWLEDGMENTS}

The authors thank W. Vogelsang for the LHAPDF evaluation of the coupling, E. Ebadati for providing the numerical solution of the gap equation, M. Q. Huber for the Landau gauge quark propagator data, and M. Quandt for a critical reading of the manuscript. This work was supported but the Deutsche Forschungsgemeinschaft (DFG) under Contract No. DFG-Re856/10-1.

\section{APPENDIX A: COHERENT-STATE REPRESENTATION OF FERMION FIELDS}

The coherent-state representation of the fermionic Fock space has been introduced in Ref. [31] in coordinate space. For the sake of completeness we collect here the relevant results in momentum space. The Dirac field $\psi$ is expanded in the usual way $\psi(\mathbf{x})=\int \frac{\mathrm{d}^{3} p}{(2 \pi)^{3}} \mathrm{e}^{\mathrm{i} \cdot \mathbf{x}} \psi(\mathbf{p})$,

$\psi(\mathbf{p})=\frac{1}{\sqrt{2 E_{\mathbf{p}}}}\left[u(\mathbf{p}, s) b(\mathbf{p}, s)+v(-\mathbf{p}, s) d^{\dagger}(-\mathbf{p}, s)\right]$

in terms of the eigenspinors $u(\mathbf{p}, s), v(\mathbf{p}, s)$ of the free Dirac Hamiltonian $h_{0}(\mathbf{p})$ [Eq. (6)] satisfying the eigenvalue equations

$h_{0}(\mathbf{p}) u(\mathbf{p}, s)=E_{\mathbf{p}} u(\mathbf{p}, s), \quad h_{0}(\mathbf{p}) v(-\mathbf{p}, s)=-E_{\mathbf{p}} v(-\mathbf{p}, s)$,

where $s= \pm 1$ accounts for the two spin degrees of freedom. With the usual normalization the Dirac eigenspinors satisfy the orthonormality relations

$$
\begin{aligned}
u^{\dagger}(\mathbf{p}, s) u\left(\mathbf{p}, s^{\prime}\right) & =2 E_{\mathbf{p}} \delta_{s s^{\prime}}=v^{\dagger}(\mathbf{p}, s) v\left(\mathbf{p}, s^{\prime}\right), \\
u^{\dagger}(\mathbf{p}, s) \beta u\left(\mathbf{p}, s^{\prime}\right) & =2 m \delta_{s s^{\prime}}=-v^{\dagger}(\mathbf{p}, s) \beta v\left(\mathbf{p}, s^{\prime}\right), \\
u^{\dagger}(\mathbf{p}, s) v\left(-\mathbf{p}, s^{\prime}\right) & =0 .
\end{aligned}
$$

The expansion coefficients $b(\mathbf{p}, s), d^{\dagger}(\mathbf{p}, s)$ are annihilation and creation operators satisfying the usual anticommutation relations

$$
\left\{b(\mathbf{p}, s), b^{\dagger}(\mathbf{q}, t)\right\}=\delta_{s t}(2 \pi)^{3} \delta(\mathbf{p}-\mathbf{q})=\left\{d(\mathbf{p}, s), d^{\dagger}(\mathbf{q}, t)\right\},
$$

which, with the normalization (A2), ensure that the Fermi field in coordinate space has the canonical anticommutation relation

$$
\left\{\psi(\mathbf{x}), \psi^{\dagger}(\mathbf{y})\right\}=\delta(\mathbf{x}-\mathbf{y}) .
$$

Furthermore, the operators $b(\mathbf{p}, s)$ and $d(\mathbf{p}, s)$ annihilate the filled Dirac sea of the free fermions denoted by $|0\rangle$, i.e.

$$
b(\mathbf{p}, s)|0\rangle=0=d(\mathbf{p}, s)|0\rangle .
$$

The eigenspinors $u$ and $v$ are also eigenvalues of the projectors [Eq. (5)]

$$
\begin{aligned}
\Lambda_{+}(\mathbf{p}) u(\mathbf{p}, s) & =u(\mathbf{p}, s), & \Lambda_{+}(\mathbf{p}) v(-\mathbf{p}, s) & =0, \\
\Lambda_{-}(\mathbf{p}) v(-\mathbf{p}, s) & =v(-\mathbf{p}, s), & \Lambda_{-}(\mathbf{p}) u(\mathbf{p}, s) & =0 .
\end{aligned}
$$

Furthermore, the projectors $\Lambda_{ \pm}$are related to the Dirac spinors by the following completeness relations:

$$
\begin{aligned}
\sum_{s} \frac{u(\mathbf{p}, s) \otimes u^{\dagger}(\mathbf{p}, s)}{2 E_{\mathbf{p}}} & =\Lambda_{+}(\mathbf{p}), \\
\sum_{s} \frac{v(-\mathbf{p}, s) \otimes v^{\dagger}(-\mathbf{p}, s)}{2 E_{\mathbf{p}}} & =\Lambda_{-}(\mathbf{p}) .
\end{aligned}
$$

Since we have two sets of fermion operators $b, b^{\dagger}$ and $d, d^{\dagger}$, corresponding to particles and antiparticles, we need also 
two different sets of Grassmann variables. Given the decomposition [Eq. (A1)] of the Dirac field it is convenient to define the coherent fermion states $\left|\xi_{+}, \xi_{-}^{*}\right\rangle$ of the Dirac fermions by

$$
\begin{aligned}
b(\mathbf{p}, s)\left|\xi_{+}, \xi_{-}^{*}\right\rangle & =\xi_{+}(\mathbf{p}, s)\left|\xi_{+}, \xi_{-}^{*}\right\rangle, \\
d(\mathbf{p}, s)\left|\xi_{+}, \xi_{-}^{*}\right\rangle & =\xi_{-}^{*}(\mathbf{p}, s)\left|\xi_{+}, \xi_{-}^{*}\right\rangle,
\end{aligned}
$$

and to introduce the Grassmann-valued Dirac spinor fields

$$
\begin{gathered}
\xi_{+}(\mathbf{p}):=\frac{1}{\sqrt{2 E_{\mathbf{p}}}} \sum_{s} u(\mathbf{p}, s) \xi_{+}(\mathbf{p}, s), \\
\xi_{-}^{\dagger}(\mathbf{p}):=\frac{1}{\sqrt{2 E_{\mathbf{p}}}} \sum_{s} v^{\dagger}(-\mathbf{p}, s) \xi_{-}^{*}(-\mathbf{p}, s),
\end{gathered}
$$

which satisfy

$$
\Lambda_{ \pm}(\mathbf{p}) \xi_{ \pm}(\mathbf{p})=\xi_{ \pm}(\mathbf{p}) .
$$

From Eq. (A2) follow the inverse relations to Eq. (A3)

$$
\begin{aligned}
& \xi_{+}(\mathbf{p}, s)=\frac{1}{\sqrt{2 E_{\mathbf{p}}}} u^{\dagger}(\mathbf{p}, s) \xi_{+}(\mathbf{p}), \\
& \xi_{-}^{*}(\mathbf{p}, s)=\frac{1}{\sqrt{2 E_{\mathbf{p}}}} \xi_{-}^{\dagger}(\mathbf{p}) v^{\dagger}(-\mathbf{p}, s) .
\end{aligned}
$$

For simplicity we will simply write $|\xi\rangle$ instead of $\left|\xi_{+}, \xi_{-}^{*}\right\rangle$. With these definitions we find

$$
\begin{aligned}
\frac{u^{\dagger}(\mathbf{p}, s)}{\sqrt{2 E_{\mathbf{p}}}}\langle\xi| b^{m \dagger}(\mathbf{p}, s) & =\xi_{+}^{m^{\dagger}}(\mathbf{p})\langle\xi| \\
\frac{u(\mathbf{p}, s)}{\sqrt{2 E_{\mathbf{p}}}}\langle\xi| b^{m}(\mathbf{p}, s) & =\frac{\delta}{\delta_{-}^{m^{\dagger}}(\mathbf{p})}\langle\xi| \\
\frac{v(-\mathbf{p}, s)}{\sqrt{2 E_{\mathbf{p}}}}\langle\xi| d^{m^{\dagger}}(-\mathbf{p}, s) & =\xi_{-}^{m}(\mathbf{p})\langle\xi| \\
\frac{v^{\dagger}(-\mathbf{p}, s)}{\sqrt{2 E_{\mathbf{p}}}}\langle\xi| d^{m}(-\mathbf{p}, s) & =\frac{\delta}{\delta \xi_{-}^{m}(\mathbf{p})}\langle\xi| .
\end{aligned}
$$

Furthermore, the coherent-state representation of a Fock state $|\Phi\rangle$ of the Dirac fermions is given by

$$
\Phi\left[\xi_{+}^{\dagger}, \xi_{-}\right]=\langle\xi \mid \Phi\rangle, \quad \Phi^{*}\left[\xi_{+}, \xi_{-}^{\dagger}\right]=\langle\Phi \mid \xi\rangle .
$$

In the following it will be also convenient to assemble the independent fields $\xi_{+}$and $\xi_{-}$in a single Grassmann-valued spinor

$$
\xi(\mathbf{p})=\xi_{+}(\mathbf{p})+\xi_{-}(\mathbf{p}), \quad \xi_{ \pm}(\mathbf{p})=\Lambda_{ \pm}(\mathbf{p}) \xi(\mathbf{p}) .
$$

In analogy to the Fourier decomposition (A1) of the Fermi field we also introduce the Grassmann fields in the coordinate representation

$$
\xi_{ \pm}(\mathbf{x})=\int \frac{\mathrm{d}^{3} p}{(2 \pi)^{3}} \mathrm{e}^{\mathbf{i} \mathbf{p} \cdot \mathbf{x}} \xi_{ \pm}(\mathbf{p}),
$$

which implies

$$
\xi(\mathbf{x})=\xi_{+}(\mathbf{x})+\xi_{-}(\mathbf{x})
$$

and

$$
\frac{\delta}{\delta \xi_{ \pm}(\mathbf{x})}=\int \frac{\mathrm{d}^{3} p}{(2 \pi)^{3}} \mathrm{e}^{-\mathrm{i} \mathbf{p} \cdot \mathbf{x}} \frac{\delta}{\delta \xi_{ \pm}(\mathbf{p})} .
$$

From Eq. (A4) then follows that the action of the Fermi field $\psi(\mathbf{x})\left[\right.$ Eq. (A1)] on the coherent state $|\xi\rangle \equiv\left|\xi_{+}, \xi_{-}^{*}\right\rangle$ is given by

$$
\begin{aligned}
\langle\xi| \psi(\mathbf{x}) & =\left(\xi_{-}(\mathbf{x})+\frac{\delta}{\delta \xi_{+}^{\dagger}(\mathbf{x})}\right)\langle\xi|, \\
\langle\xi| \psi^{\dagger}(\mathbf{x}) & =\left(\xi_{+}^{\dagger}(\mathbf{x})+\frac{\delta}{\delta \xi_{-}(\mathbf{x})}\right)\langle\xi| .
\end{aligned}
$$

\section{APPENDIX B: THE QUARK CRDSE}

In the bare-vertex approximation the CRDSE (25) for the quark propagator Eq. (24) reduces in the chiral limit to the following set of equations for the dressing functions:

$$
\begin{aligned}
A_{p}= & 1+\frac{g^{2} C_{F}}{2} \int \frac{\mathrm{d}^{3} q}{(2 \pi)^{3}} \frac{1}{\Omega(\mathbf{p}+\mathbf{q}) \Delta_{q}}\left\{A_{q}\left[X_{-}(\mathbf{p}, \mathbf{q}) \frac{|V(\mathbf{p}, \mathbf{q})|^{2}+|V(\mathbf{q}, \mathbf{p})|^{2}}{2}+X_{+}(\mathbf{p}, \mathbf{q}) \frac{|W(\mathbf{p}, \mathbf{q})|^{2}+|W(\mathbf{q}, \mathbf{p})|^{2}}{2}\right]\right. \\
& \left.+D_{q}\left[X_{-}(\mathbf{p}, \mathbf{q}) \frac{|V(\mathbf{p}, \mathbf{q})|^{2}-|V(\mathbf{q}, \mathbf{p})|^{2}}{2}+X_{+}(\mathbf{p}, \mathbf{q}) \frac{|W(\mathbf{p}, \mathbf{q})|^{2}-|W(\mathbf{q}, \mathbf{p})|^{2}}{2}\right]\right\} \\
B_{p}= & \Re s_{p}+\frac{g^{2} C_{F}}{2} \int \frac{\mathrm{d}^{3} q}{(2 \pi)^{3}} \frac{1}{\Omega(\mathbf{p}+\mathbf{q}) \Delta_{q}}\left\{B_{q}\left[X_{-}(\mathbf{p}, \mathbf{q}) \Re[V(\mathbf{p}, \mathbf{q}) V(\mathbf{q}, \mathbf{p})]-X_{+}(\mathbf{p}, \mathbf{q}) \Re[W(\mathbf{p}, \mathbf{q}) W(\mathbf{q}, \mathbf{p})]\right]\right. \\
& \left.+C_{q}\left[X_{-}(\mathbf{p}, \mathbf{q}) \Im[V(\mathbf{p}, \mathbf{q}) V(\mathbf{q}, \mathbf{p})]-X_{+}(\mathbf{p}, \mathbf{q}) \Im[W(\mathbf{p}, \mathbf{q}) W(\mathbf{q}, \mathbf{p})]\right]\right\},
\end{aligned}
$$




$$
\begin{aligned}
C_{p}= & \Im s_{p}+\frac{g^{2} C_{F}}{2} \int \frac{\mathrm{d}^{3} q}{(2 \pi)^{3}} \frac{1}{\Omega(\mathbf{p}+\mathbf{q}) \Delta_{q}}\left\{-C_{q}\left[X_{-}(\mathbf{p}, \mathbf{q}) \Re[V(\mathbf{p}, \mathbf{q}) V(\mathbf{q}, \mathbf{p})]-X_{+}(\mathbf{p}, \mathbf{q}) \Re[W(\mathbf{p}, \mathbf{q}) W(\mathbf{q}, \mathbf{p})]\right]\right. \\
& \left.+B_{q}\left[X_{-}(\mathbf{p}, \mathbf{q}) \Im[V(\mathbf{p}, \mathbf{q}) V(\mathbf{q}, \mathbf{p})]-X_{+}(\mathbf{p}, \mathbf{q}) \Im[W(\mathbf{p}, \mathbf{q}) W(\mathbf{q}, \mathbf{p})]\right]\right\}, \\
D_{p}= & \frac{g^{2} C_{F}}{2} \int \frac{\mathrm{d}^{3} q}{(2 \pi)^{3}} \frac{1}{\Omega(\mathbf{p}+\mathbf{q}) \Delta_{q}}\left\{D_{q}\left[X_{-}(\mathbf{p}, \mathbf{q}) \frac{|V(\mathbf{p}, \mathbf{q})|^{2}+|V(\mathbf{q}, \mathbf{p})|^{2}}{2}+X_{+}(\mathbf{p}, \mathbf{q}) \frac{|W(\mathbf{p}, \mathbf{q})|^{2}+|W(\mathbf{q}, \mathbf{p})|^{2}}{2}\right]\right. \\
& \left.+A_{q}\left[X_{-}(\mathbf{p}, \mathbf{q}) \frac{|V(\mathbf{p}, \mathbf{q})|^{2}-|V(\mathbf{q}, \mathbf{p})|^{2}}{2}+X_{+}(\mathbf{p}, \mathbf{q}) \frac{|W(\mathbf{p}, \mathbf{q})|^{2}-|W(\mathbf{q}, \mathbf{p})|^{2}}{2}\right]\right\},
\end{aligned}
$$

where $\Delta_{q}$ is given by Eq. (33) and $X_{ \pm}(\mathbf{p}, \mathbf{q})$ by Eq. (46).

\section{APPENDIX C: THE VACUUM ENERGY DENSITY}

When the full quark-gluon vertex $\bar{\Gamma}$ [Eq. (21)] is replaced by the bare one $\bar{\Gamma}_{0}$ [Eq. (13), (18)], the remaining traces in the energy density contributions [Eqs. (27) and (28)] can be worked out explicitly. One finds for the second piece of the singleparticle Hamiltonian equation (27)

$$
\begin{aligned}
e_{\mathrm{D}}^{(1)}= & g^{2} C_{F} \int \frac{\mathrm{d}^{3} q}{(2 \pi)^{3}} \frac{\mathrm{d}^{3} \ell}{(2 \pi)^{3}} \frac{X_{-}(\mathbf{q}, \boldsymbol{\ell})}{\Delta_{q} \Delta_{\ell} \Omega(\mathbf{q}+\boldsymbol{\ell})}\left\{\left[\left(A_{q}-D_{q}\right)\left(A_{\ell}+D_{\ell}\right)+B_{q} B_{\ell}-C_{q} C_{\ell}\right] \Re V(\mathbf{q}, \boldsymbol{\ell})+\left[B_{q} C_{\ell}+C_{q} B_{\ell}\right] \Im V(\mathbf{q}, \boldsymbol{\ell})\right\} \\
& +g^{2} C_{F} \int \frac{\mathrm{d}^{3} q}{(2 \pi)^{3}} \frac{\mathrm{d}^{3} \ell}{(2 \pi)^{3}} \frac{X_{+}(\mathbf{q}, \boldsymbol{\ell})}{\Delta_{q} \Delta_{\ell} \Omega(\mathbf{q}+\boldsymbol{\ell})}\left\{\left[\left(A_{q}-D_{q}\right) B_{\ell}+\left(A_{\ell}+D_{\ell}\right) B_{q}\right] \Re W(\mathbf{q}, \boldsymbol{\ell})\right. \\
& \left.+\left[\left(A_{q}-D_{q}\right) C_{\ell}+\left(A_{\ell}+D_{\ell}\right) C_{q}\right] \Im W(\mathbf{q}, \boldsymbol{\ell})\right\}
\end{aligned}
$$

with $X_{ \pm}(\mathbf{q}, \boldsymbol{\ell})$ given in Eq. (46). Furthermore, the contribution equation (28) from the kinetic energy of the gluons reduces to

$$
e_{E}^{q}=g^{2} C_{F} \int \frac{\mathrm{d}^{3} q}{(2 \pi)^{3}} \frac{\mathrm{d}^{3} \ell}{(2 \pi)^{3}} \frac{A_{q}+D_{q}}{\Delta_{q}} \frac{A_{\ell}+D_{\ell}}{\Delta_{\ell}}\left\{X_{-}(\mathbf{q}, \boldsymbol{\ell})|V(\mathbf{q}, \boldsymbol{\ell})|^{2}+X_{+}(\mathbf{q}, \boldsymbol{\ell})|W(\mathbf{q}, \boldsymbol{\ell})|^{2}\right\} .
$$

\section{APPENDIX D: THE QUARK GAP EQUATION}

In the bare-vertex approximation one finds from the minimization of the energy density for the scalar kernel $s_{p}$ the following equation:

$$
\begin{aligned}
|\mathbf{p}| s_{p}= & \frac{g^{2} C_{F}}{2} \int \frac{\mathrm{d}^{3} q}{(2 \pi)^{3}} \frac{1}{\left(1+s_{q}^{2}\right) \Omega(\mathbf{p}+\mathbf{q})}\left\{X_{-}(\mathbf{p}, \mathbf{q}) V(\mathbf{p}, \mathbf{q})\left[\left(1-s_{p}^{2}\right) s_{q}-2 s_{p}\right)\right]+X_{+}(\mathbf{p}, \mathbf{q}) W(\mathbf{p}, \mathbf{q})\left[1-s_{p}^{2}-2 s_{p} s_{q}\right] \\
& -\frac{|\mathbf{p}|}{1+s_{p}^{2}}\left[X_{-}(\mathbf{p}, \mathbf{q}) V^{2}(\mathbf{p}, \mathbf{q})\left[s_{p}\left(s_{p}^{2}-3\right)+s_{q}\left(1-3 s_{p}^{2}\right)\right]+X_{+}(\mathbf{p}, \mathbf{q}) W^{2}(\mathbf{p}, \mathbf{q})\left[s_{p}\left(s_{p}^{2}-3\right)-s_{q}\left(1-3 s_{p}^{2}\right)\right]\right] \\
& \left.-\frac{|\mathbf{q}|}{1+s_{q}^{2}}\left[X_{-}(\mathbf{p}, \mathbf{q}) V^{2}(\mathbf{p}, \mathbf{q})\left[\left(1-s_{p}^{2}\right) s_{q}-s_{p}\left(1-s_{q}^{2}\right)\right]-X_{+}(\mathbf{p}, \mathbf{q}) W^{2}(\mathbf{p}, \mathbf{q})\left[\left(1-s_{p}^{2}\right) s_{q}+s_{p}\left(1-s_{q}^{2}\right)\right]\right]\right\} \\
& +\frac{g^{2} C_{F}}{2} \int \frac{\mathrm{d}^{3} q}{(2 \pi)^{3}} \frac{s_{p}}{1+s_{q}^{2}}\left[X_{-}(\mathbf{p}, \mathbf{q}) V^{2}(\mathbf{p}, \mathbf{q})+X_{+}(\mathbf{p}, \mathbf{q}) W^{2}(\mathbf{p}, \mathbf{q})\right] \\
& +\frac{g^{2} C_{F}}{2} \int \frac{\mathrm{d}^{3} q}{(2 \pi)^{3}} \frac{F(\mathbf{p}-\mathbf{q})}{1+s_{q}^{2}}\left[s_{q}\left(1-s_{p}^{2}\right)-\hat{\mathbf{p}} \cdot \hat{\mathbf{q}}\left(1-s_{p}^{2}\right)\right]
\end{aligned}
$$


[1] S. Aoki et al., Eur. Phys. J. C 77, 112 (2017).

[2] M. Faber and R. Höllwieser, Prog. Part. Nucl. Phys. 97, 312 (2017).

[3] R. Alkofer and L. von Smekal, Phys. Rep. 353, 281 (2001).

[4] J. M. Pawlowski, Ann. Phys. (Amsterdam) 322, 2831 (2007).

[5] C. S. Fischer, J. Phys. G 32, R253 (2006).

[6] D. Binosi and J. Papavassiliou, Phys. Rep. 479, 1 (2009).

[7] G. Eichmann, H. Sanchis-Alepuz, R. Williams, R. Alkofer, and C. S. Fischer, Prog. Part. Nucl. Phys. 91, 1 (2016).

[8] C. Feuchter and H. Reinhardt, Phys. Rev. D 70, 105021 (2004); H. Reinhardt and C. Feuchter, Phys. Rev. D 71, 105002 (2005).

[9] D. Epple, H. Reinhardt, and W. Schleifenbaum, Phys. Rev. D 75, 045011 (2007).

[10] P. Vastag, H. Reinhardt, and D. Campagnari, Phys. Rev. D 93, 065003 (2016).

[11] Y. Nambu, Phys. Rev. D 10, 4262 (1974).

[12] G. 't Hooft, Nucl. Phys. B190, 455 (1981).

[13] G. Mack and V. B. Petkova, Ann. Phys. (N.Y.) 123, 442 (1979).

[14] H. B. Nielsen and P. Olesen, Nucl. Phys. B160, 380 (1979).

[15] L. D. Debbio, M. Faber, J. Giedt, J. Greensite, and S. Olejnik, Phys. Rev. D 58, 094501 (1998).

[16] K. Langfeld, H. Reinhardt, and O. Tennert, Phys. Lett. B 419, 317 (1998).

[17] V. Gribov, Nucl. Phys. B139, 1 (1978).

[18] D. Zwanziger, Nucl. Phys. B518, 237 (1998).

[19] J. Greensite, S. Olejnik, and D. Zwanziger, J. High Energy Phys. 05 (2005) 070.

[20] H. Reinhardt, Phys. Rev. Lett. 101, 061602 (2008).

[21] G. Burgio, M. Quandt, H. Reinhardt, and H. Vogt, Phys. Rev. D 92, 034518 (2015).

[22] J. R. Finger and J.E. Mandula, Nucl. Phys. B199, 168 (1982).

[23] A. Amer, A. Le Yaouanc, L. Oliver, O. Pene, and J.c. Raynal, Phys. Rev. Lett. 50, 87 (1983).

[24] A. Le Yaouanc, L. Oliver, O. Pene, and J. C. Raynal, Phys. Rev. D 29, 1233 (1984).
[25] S. L. Adler and A. Davis, Nucl. Phys. B244, 469 (1984).

[26] P. J. d. A. Bicudo and J. E. F. T. Ribeiro, Phys. Rev. D 42, 1611 (1990).

[27] R. Alkofer and P. Amundsen, Nucl. Phys. B306, 305 (1988).

[28] M. Pak and H. Reinhardt, Phys. Lett. B 707, 566 (2012); Phys. Rev. D 88, 125021 (2013).

[29] D. R. Campagnari, E. Ebadati, H. Reinhardt, and P. Vastag, Phys. Rev. D 94, 074027 (2016).

[30] D. R. Campagnari and H. Reinhardt, Phys. Rev. D 82, 105021 (2010).

[31] D. R. Campagnari and H. Reinhardt, Phys. Rev. D 92, 065021 (2015).

[32] N. H. Christ and T. D. Lee, Phys. Rev. D 22, 939 (1980).

[33] P. Watson and H. Reinhardt, Phys. Rev. D 85, 025014 (2012).

[34] G. Burgio, M. Schrock, H. Reinhardt, and M. Quandt, Phys. Rev. D 86, 014506 (2012).

[35] M. Pak and M. Schröck, Phys. Rev. D 91, 074515 (2015).

[36] D. R. Campagnari and H. Reinhardt, Int. J. Mod. Phys. A 30, 1550100 (2015).

[37] C. Popovici, P. Watson, and H. Reinhardt, Phys. Rev. D 79, 045006 (2009).

[38] A. Buckley, J. Ferrando, S. Lloyd, K. Nordström, B. Page, M. Rüfenacht, M. Schönherr, and G. Watt, Eur. Phys. J. C 75, 132 (2015).

[39] Y. Nakagawa, A. Nakamura, T. Saito, H. Toki, and D. Zwanziger, Phys. Rev. D 73, 094504 (2006).

[40] M. Golterman, J. Greensite, S. Peris, and A. P. Szczepaniak, Phys. Rev. D 85, 085016 (2012).

[41] J. Greensite and A. P. Szczepaniak, Phys. Rev. D 91, 034503 (2015).

[42] S. Borsanyi, S. Dürr, Z. Fodor, S. Krieg, A. Schäfer, E. E. Scholz, and K. K. Szabó, Phys. Rev. D 88, 014513 (2013).

[43] G. Cossu, H. Fukaya, S. Hashimoto, T. Kaneko, and J.-I. Noaki, Prog. Theor. Exp. Phys. 2016, 093 B06 (2016).

[44] M. Tomii, G. Cossu, B. Fahy, H. Fukaya, S. Hashimoto, T. Kaneko, and J. Noaki (JLQCD Collaboration), Phys. Rev. D 96, 054511 (2017). 